\title{
Perception et comportement des hommes, des femmes, et des prestataires des services en matiére des morbidités relatives a la santé de la reproduction
}

Direction de la Santé et de la Famille (DSF)

University of Ouagadougou Demographic Research Unit (UERD)

Mwangaza

Population Council

Follow this and additional works at: https://knowledgecommons.popcouncil.org/departments_sbsr-rh How does access to this work benefit you? Let us know!

\section{Recommended Citation}

"Perception et comportement des hommes, des femmes, et des prestataires des services en matiére des morbidités relatives a la santé de la reproduction," Final report. Washington, DC and Bazega: Population Council and Laboratoire de Santé Communautaire, 1998. 


\section{MINISTERE DE LA SANTE}

Secrétariat Général

Direction Générale de la Santé Publique

Direction de la Santé

Unité d'Enseignement et de Recherche

Mwangaza en Démographie
Population Council Africa OR/TA II

\section{Laboratoire de Sante Communautaire,} BAZEGA

PERCEPTION ET COMPORTEMENT DES HOMMES, DES FEMMES ET DES PRESTATAIRES DE SERVICES EN MATIERE DE MORBIDITES RELATIVES A LA SANTE DE LA REPRODUCTION

Ouagadougou, Burkina Faso

Juin 1998 


\section{TABLE DES MATIERES}

RESUME

.iii

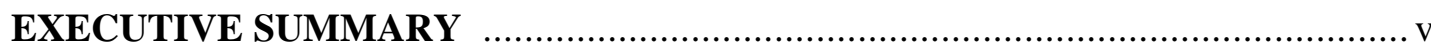

I. INTRODUCTION A L'ETUDE

1. Contexte et identification du problème

2. Justification de l'étude

3. Objectifs de l'étude

4. Définitions des concepts

5. Approche méthodologique

II. RESULTATS .5

1. Au niveau de la communauté

1.1 Les infections sexuellement transmissibles : maladies et symptômes

1.2 La prise en charge et le traitement des personnes malades par la communauté

1.3 L'itinéraire thérapeutique

1.4 La prévention des IGP/IST

1.5 La communication et les interactions conjugales en matière d'IGP/IST

2. Au niveau des prestataires professionnels et communautaires

2.1 Population et ampleur des pathologies génitales

2.2 Problèmes et conséquences des IGP/IST non traitées

2.3 Traitement et prévention

2.4 La communication autour des IGP/IST

2.5 Propositions pour une amélioration de la prise en charge des clients

III. CONCLUSION ET RECOMMANDATIONS ….......................................... 18

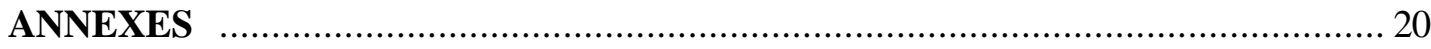

A. Vocabulaire utilisé par la communauté pour désigner les morbidités de la reproduction

B. Détails sur l'approche méthodologique et les difficultés rencontrées

C. Détail descriptif des IGP/IST par les prestataires

D. Quelques propos retenus

E. Résultats du tri par piles 


\section{TABLEAU DES SIGLES ET ABREVIATIONS}

- ASC

- CIPD

- CNLS

- $\mathrm{DBC}$

- DSF

- EIA

- FS

- GDD

- IEC

- IGP

- INA

- IST

- ITUG

- LSC

- MGF

- NESCAFE

- OMS

- $\mathrm{SMI} / \mathrm{PF}$

- SOPAL

- SRO

- VIH/SIDA
Agent de santé communautaire

Conférence internationale sur la population et le développement

Comité national de lutte contre le SIDA

Distribution à base communautaire (agent)

Direction de la santé de la famille

Entretien individuel approfondi

Formation sanitaire

Groupe de discussion dirigée

Information, éducation, communication

Infection génito-pelvienne

Institut national d'alphabétisation

Infection sexuellement transmissible

Infection du tractus uro-génital

Laboratoire de santé communautaire

Mutilation génitale féminine

Café du Groupe NESTLE

Organisation mondiale de la santé

Santé maternelle et infantile/planification familiale

Société de production d'alcool

Sel de réhydratation orale

Virus du syndrome immuno déficitaire acquis/syndrome immuno déficitaire acquis 


\section{RESUME}

Cette étude vient à point nommé pour fournir des informations sur le vocabulaire utilisé pour désigner les infections pelviennes et génitales et les infections sexuellement transmissibles (IST). Les prestataires et le Ministère de la Santé ainsi que tout intervenant pourront par conséquent utiliser les résultats pour l'élaboration de messages IEC appropriés.

Aussi bien en ville qu'en campagne, la nécessité d'avoir une meilleure compréhension de la perception et de la définition des diverses morbidités de la reproduction et de leurs symptômes, l'itinéraire thérapeutique ou la prise en charge médicale ou communautaire, tant au niveau de la communauté ellemême qu'au niveau des prestataires, est primordiale. C'est dans cet esprit qu'il a été commandité une étude qualitative pour contribuer à l'élaboration d'interventions communautaires et cliniques appropriées dans la prise en charge des IST/VIH par la communauté et par les prestataires.

La présente étude a nécessité l'utilisation de quatre approches méthodologiques en recherche qualitative pour la collecte et l'analyse des données dans les zones A et B du LSC. Ce sont les groupes de discussions dirigées, les entretiens individuels approfondis, le listage libre et le tri par piles. Au niveau de la communauté, cinq groupes cibles étaient concernés : les filles célibataires de moins de 25 ans, les femmes mariées de 25 à 39 ans, les femmes de plus de 40 ans, les hommes célibataires de moins de 25 ans, et les hommes mariés de 25 à 39 ans. Au niveau des prestataires, l'étude s'est intéressée aux prestataires professionnels (clinique) et aux prestataires non professionnels (communautaire : agents de santé communautaire et agents de distribution à base communautaire) avec lesquels des entretiens individuels approfondis ont été réalisés.

\section{Résultats clés au niveau de la communauté}

Tous les groupes interrogés utilisent des termes et expressions spécifiques locaux pour désigner les IST. Les termes les plus évoqués sont le «soumpiisi » pour désigner la gonococcie et le «tuma» pour désigner la syphilis. Les principaux symptômes notés sont les douleurs mictionnelles, les lésions, les plaies, et les boutons chez l'homme ou chez la femme. Quant aux causes, elles sont soit traditionnelles, supposées ou réelles ou encore inconnues.

Cependant le niveau de connaissance de ces infections varie selon la tranche d'âge et le sexe. Le VIH est par ailleurs bien connu par tous, surtout en ce qui concerne les principaux symptômes. Quant aux conséquences des IST non traitées il est souvent cité : la mort, la stérilité, la marginalisation et la destruction des organes génitaux.

La prise en charge des malades est comprise de plusieurs façons. D'abord, certains fatalistes renvoient le malade à la prière tout simplement pour obtenir guérison. La qualification de maladie se rapportant à tel ou tel sexe (" maladie des femmes » ou « maladie des hommes ») quoique rare, est quand même évoquée. Cependant et pour la plupart des propos entendus lors de la collecte, il ressort que la conduite du malade à la formation sanitaire et le fait d'aider celui-ci à trouver un traitement approprié sont les deux voies prioritaires de la communauté.

Même si d'une part, le guérisseur traditionnel, le prestataire clinique ou communautaire sont consultés et d'autre part, la prière et l'automédication sont employés de façon courante au sein de la communauté, il est à noter que pour tous les groupes, le «meilleur traitement est celui qui apporte la guérison ». Pour prévenir toute infection, il est recommandé en priorité d'utiliser la capote, d'être fidèle, de suivre les conseils des prestataires et d'accepter le traitement des partenaires.

La gêne et la honte font qu'il est souvent difficile aux gens de parler entre eux ou avec les prestataires, des IST. Les discussions ont lieu surtout lorsque le mal a atteint un niveau considérable (insupportable, visible, douloureux). Cependant, lorsqu'elles ont lieu, elles se déroulent entre hommes, entre femmes, entre ami(e)s, de fille à mère ou de nièce à tante. En général, l'homme a plus de facilité à parler d'une 
IST dont il souffre. Par contre, la femme se doit d'obtenir non seulement l'autorisation d'aller en consultation, mais aussi elle a souvent recours au mari pour l'achat des médicaments. Pour cela, le rôle du prestataire est primordial car il atténue les disputes et conséquences souvent catastrophiques pour le couple. Une telle affirmation s'observe chez les femmes mariées notamment.

\section{Résultats clés au niveau des prestataires}

Ils reconnaissent unanimement que les infections pelviennes et notamment les IST sont fréquentes dans leur aire et causent de multiples dégâts. Les hommes et les femmes ayant une vie sexuelle active sont les plus touchés. Les conséquences de telles infections sont observables par les deux types de prestataires à plusieurs niveaux : d'abord sur le plan médical, ensuite sur le plan social et enfin sur le plan économique.

Tandis que les prestataires professionnels perçoivent leur rôle dans un cadre global de traitement curatif et préventif, les autres, c'est-à-dire les prestataires communautaires, le perçoivent pour la plupart dans la prévention, la conscientisation de leur communauté d'une part sur l'existence réelle des maladies, mais aussi sur leurs conséquences. Tous préconisent cependant l'abstinence, la fidélité et l'utilisation des préservatifs comme mesures préventives contre les IST.

Pour le cas spécifique du SIDA, deux tendances se dégagent : ceux qui ne peuvent rien faire ou qui ne savent rien faire pour une personne atteinte du SIDA; et ceux qui compatissent aux douleurs (physique ou morale) du malade. Le constat général est l'insuffisance dans les acquis en matière de prise en charge des IST en général et du SIDA en particulier. Pour cela, ils attendent d'être formés ou recyclés mais aussi de bénéficier de dotation en matériel adéquat.

Même si pour la quasi-totalité des prestataires, ils sont disposés à parler à leurs clients de toutes les IST, il reste que la communauté est peu ouverte à de telles discussions. Par ailleurs, un problème de maniement de la langue locale pour exprimer les faits relatifs aux IST se pose de façon considérable.

\section{Conclusion et recommandations}

La présente étude constitue un outil de travail approprié tant pour les décideurs que pour les prestataires (clinique ou communautaire). Les efforts sont à poursuivre au niveau de la communauté pour une meilleure perception et la distillation de la bonne information sur les causes et conséquences des IST en général et du SIDA en particulier. Le caractère honteux et la gêne qui entourent les IST et le SIDA en particulier rendent leur prise en charge encore difficile. Si la guérison est l'objectif visé par le malade, il reste que les voies l'y menant sont multiples.

L'urgence de poursuivre la sensibilisation pour tous les groupes cibles s'est manifestée tout au long de l'étude. Un fait frappant est le niveau important de l'ignorance des IST et de leurs conséquences sur la vie future des filles de moins de 25 ans. Pour une telle catégorie de la population, il est suggéré la réflexion et la mise en place d'un programme de services de santé de la reproduction qui leur est destiné en même temps qu'aux garçons en milieu rural et semi-rural.

Si les prestataires professionnels ont besoin d'une formation ou d'un recyclage en IST/SIDA, les autres attendent en plus d'une formation en IST/SIDA, une dotation en matériel d'IEC. Pour tous, l'intégration des services de prise en charge des IST/SIDA et l'équipement matériel des formations sanitaires sont les bienvenus. 


\section{EXECUTIVE SUMMARY}

This study provides information on the vocabulary used to refer to reproductive tract infections and sexually transmitted infections (STIs) by the community of Bazèga, a rural province in Burkina Faso. Providers and the Ministry of Health, as well as others who wish to design interventions, will use the results to design appropriate and effective IEC materials.

In urban as well as rural areas, a need exists for a stronger understanding of communities' perceptions and definitions of reproductive tract infections and their symptoms, as well as how, when and where people treat these infections. This qualitative study was undertaken to help gain such an understanding, thus paving the way for appropriate interventions targetting providers and the community at large to effectively prevent and treat STIs.

The study used four qualitative methodological tools to collect data in the experimental cells of a larger Operations Research project implemented by the field station, Laboratoire de la santé communautaire (LSC) in Bazèga: focus group discussions, in-depth interviews, free-listing and pile sorting. Focus group discussions and in-depth interviews were held at the community level by five age/sex groups-young single women under 25, married women between 25 and 39, women over 40, young single men under 25, and married men between 25 and 39. In-depth interviews were held for two types of health providers-professional, or clinical, providers and non-professional, or communitybased, providers.

\section{Key findings at the community level}

All of the groups had specific terms and expressions to describe STIs in mooré, the local language. The terms most frequently cited were soumpissi, to describe gonorrhea, and tuma for syphilis. Symptoms most commonly mentioned were painful urination, lesions, sores and genital warts. No clear pattern was found for the causes of STIs-people cited traditional, supposed and sometimes real causes, or did not know the causes.

Knowledge of STIs varied by age and sex groups, with the younger generations being the least knowledgeable, particularly young girls. HIV/AIDS was well-known across all of the age and sex groups, especially in terms of related symptoms. Although misperceptions on its transmission were found, with people citing flies and mosquitoes, as well as having done something forbidden as possible causes. Consequences of not treating STIs cited by the community were death, sterility, marginilisation and the deterioration of genital organs.

Many different responses were given on how to care for those with STIs. Prayer or self-medication were mentioned by some. Although the majority would either bring an individual with an STI to a health center or help him or her to find an appropriate treatment. Whether a traditional healer, clinical provider, community health worker, prayer or self-medication were used, all groups agreed that the 'best treatment is the treatment that cures.' To prevent infections, condoms, fidelity, following provider's instructions, and accepting partners' treatments were all mentioned.

Barriers to openly discuss STIs were found-people remarked that shame and embarassment prevented them from discussing STIs with other community members as well as with providers. This shame and embarassment often leads people to wait until the STI has reached an advanced stage, i.e. is visible or unbearably painful, before seeking treatment. Discussions that do occur tend to happen between members of the same sex. In general, men find it easier to discuss STIs and symptoms with providers than do women. Women noted that not only do they often have to ask for their husband's permission to go to the health center, but they must also ask him for money to buy the prescribed medication. As well, women fear repudiation if they are the ones to be diagnosed with an STI before their spouses. The role of the provider in these cases is delicate, and married women felt that they needed the provider to intervene in the process. 


\section{Key findings among the health service providers}

Providers were aware of STIs in their region and their potential consequences. They identified men and women of reproductive age to be at risk (15-49 years). Providers divided the consequences of STIs into three categories-medical, social and economic.

Professional providers perceived their role as treatment and prevention, while community health providers tended to see their role as prevention and education/increasing awareness at the community level. All of the providers recommended abstinence, fidelity and condoms as preventive measures.

Two types of responses on how to handle AIDS cases were found-one which said nothing could be done, or the provider did not know what to do, and the other which sought to alleviate the patient's pain, either physically or emotionally. Generally providers felt they had insufficient training on how to manage STIs in general, and HIV/AIDS in particular. Providers expressed an interest in receiving training and refresher courses, as well as IEC materials and medical equipment to better enable them to manage and prevent STIs.

Providers felt they could openly discuss STIs with their clients, and that it was the members of the community who find it difficult to discuss these issues. The providers did remark, however, that language barriers exist between them and their patients, as they do not know the local vocabulary used by the community to express symptoms and infections, thus hindering the discussion of STIs.

\section{Conclusion and recommendations}

This report will be a useful tool for providers and decision makers. Efforts to improve the community's knowledge of STIs, with a special emphasis on AIDS, and on transmission in general need to be undertaken. These efforts will need to take into account the shame and embarrasment that often surround STIs. People in the community need to be given a variety of ways to access information and treatment for STIs due to the sensitive nature of these infections.

All of the age and sex groups need to be further educated, however adolescents and youth, particularly girls are most in need of this type of education. A program targetting youth for reproductive health services and education is recommended.

While both professional and non-professional providers need training and refresher courses on STIs/HIV/AIDS, the non-professional providers are also in need of appropriate IEC materials. For all providers, the integration of STI/HIV services, and the equipment and materials these services require, are eagerly awaited. 


\section{INTRODUCTION A L'ETUDE}

\section{Contexte et identification du problème}

Vers la fin de l'année 1994, environ 18 millions d'adultes et 1,5 millions d'enfants à travers le monde avaient été infectés par le $\mathrm{VIH}^{1}$ selon les estimations, tandis qu'en 1995, l'Organisation Mondiale de la Santé (OMS) estimait qu'il y avait 333 millions de cas d'infections sexuellement transmissibles (IST), soit approximativement un million d'infections par jour ${ }^{2}$. Selon les dernières estimations de l'OMS, le Burkina Faso a le $11^{\mathrm{è}}$ taux le plus élevé de prévalence du VIH dans le monde 3 .

Un facteur contribuant à ces taux élevés d'infection au VIH en Afrique sub-saharienne est la grande prévalence des autres infections sexuellement transmissibles. Il a été démontré que l'infection à certaines IST augmente les probabilités de contracter le VIH par contact sexuel. Les efforts pour améliorer le diagnostic et le traitement des IST s'avèrent prometteurs en réduisant les taux d'infection au VIH. Une étude récente en Tanzanie a montré qu'un traitement des IST réduisait l'incidence du $\mathrm{VIH}$ de $42 \%$ dans une population rurale sur une période de deux ans ${ }^{4}$.

Une façon d'améliorer le diagnostic et le traitement des IST qui a été proposée est l'incorporation de ces services dans les services existants de santé maternelle et infantile et de planification familiale (SMI/PF). Cela toucherait les femmes qui typiquement ne reçoivent pas de tels services, réduirait potentiellement l'étiquette associée à la fréquentation des cliniques traitant spécialement les IST, et servirait a répondre aux larges besoins des femmes en matière de santé de la reproduction. Suite à la Conférence Internationale sur la Population et le Développement (CIPD) tenue au Caire en 1994, il y avait un fort soutien d'un changement des services de planification familiale pour des services plus larges de santé de la reproduction. Tandis que les organisations essaient de mettre en ouvre les plans du Caire, il y a un grand intérêt et un effort dans le domaine de l'intégration des services IST dans le cadre des SMI/PF. Cependant il reste beaucoup de problèmes à résoudre en termes d'identification des moyens effectifs de gérer les IST dans ce cadre.

Afin de gérer les IST de manière effective, il est nécessaire d'avoir une meilleure compréhension de comment les membres de la communauté perçoivent et définissent diverses morbidités de la reproduction et leurs symptômes, ainsi que le lieu, le moment et la personne auprès de laquelle ils recherchent les soins de santé. Si les prestataires de soins doivent communiquer effectivement avec leurs clients, ils doivent parler le même langage. Non seulement cela a un impact sur les messages qui sont diffusés pour la prévention et le traitement, mais cela est particulièrement important pour la gestion du syndrome, qui dépend de la détection et du traitement des cas en se basant sur l'identification des signes et symptômes suggérant un IST. Pour que cette approche fonctionne de manière efficace, il est essentiel que les prestataires aient une claire compréhension de comment la communauté définit et décrit les divers symptômes, afin de pouvoir poser des questions appropriées.

Il y a eu peu d'études aussi bien en Afrique qu'ailleurs, qui aient utilisé des méthodes de recherche qualitative pour diagnostiquer les perceptions des morbidités de la reproduction. Cependant, une étude

\footnotetext{
${ }^{1}$ World Health Organisation (WHO). The Current Global Situation of the HIV/AIDS Pandemic; Survey Report of the Global Program on AIDS, WHO, 1995.

2 Adler, Michael, Susan Foster, John Riches and Hazel Slavin. « Sexual Health and Care : Sexually Transmitted Infections : Guidelines for Prevention and Treatment ». ODA Health and Population Occasional Paper, ODA 1996.

${ }^{3}$ Stein, Zena and Mervyn Susser. «Editorial AIDS. An update on the Global Dynamics ». American Journal of Public Health. 87(6) ; June 1997.

${ }^{4}$ Grosskurth, Heiner et al. «Impact of improved treatment of sexually transmitted diseases on HIV infection in rural Tanzania : randomized controlled trial ». The Lancet. 346 ; August 26, 1995.
} 
effectuée au Nigeria par Olukoya et Elias $^{5}$ a essentiellement utilisé des groupes de discussions dirigées pour examiner les perceptions des morbidités du tractus génital chez les femmes et les hommes. Les résultats révélaient l'importance de ces perceptions sur la compréhension de l'infertilité, de l'avortement et de l'utilisation de la contraception. Le milieu rural d'Egypte a été le cadre d'une étude communautaire faite par Zuryak et al. ${ }^{6}$ qui avait été entreprise en reconnaissance du fait que les études effectuées dans les hôpitaux et les formations sanitaires ont tendance à sous-estimer le problème des maladies gynécologiques.

\section{Justification de l'étude}

Depuis 1988, le Gouvernement du Burkina Faso a adopté une approche ouverte dans la prévention et la prise en charge des IST et plus particulièrement du VIH/SIDA. En 1989, le Comité national de lutte contre le SIDA (CNLS) et l'Organisation mondiale de la santé (OMS) ont élaboré un plan triennal de lutte contre le SIDA. Les activités programmées comprennent: l'information, l'éducation et la communication pour les groupes cibles; l'approvisionnement des banques de sang en toute sécurité; des services de santé de qualité aux patients; l'élaboration d'un système de séro-surveillance ; et la recherche opérationnelle. Les activités du programme impliquaient les mass média, dont la presse écrite et parlée, la télévision sur le sujet du SIDA. Du matériel d'IEC sur les IST/SIDA ont été élaborés et placés dans des points stratégiques pour sensibiliser la population.

Le programme quinquennal de 1992 a mis l'accent sur l'intégration des activités en matière de IST/VIH/SIDA et des activités de planification familiale. La mise en œuvre d'un tel programme d'intégration comprenait un programme de formation continue du personnel médical et paramédical.

Comme dans la plupart des pays de l'Afrique sub-saharienne, au Burkina Faso il manque des données sur la prévalence des IST, y compris la zone de l'étude. Les données de l'Analyse Situationnelle Nationale de 1996, les études sélectionnées et les preuves anecdotiques rapportées par les praticiens des formations sanitaires suggèrent que les IST constituent probablement un grave problème de santé publique au Burkina Faso. Par exemple, une étude menée à Bobo Dioulasso en 1995 par Meda et al. ${ }^{7}$ rapportait que $77 \%$ des 220 patientes venues pour des traitements à l'hôpital avec des symptômes d'infections des voies urinaires, souffraient effectivement de IST ; $28 \%$ d'entre elles avaient des trichomonas, $27 \%$ des chlamydia, $11 \%$ la gonorrhée et $42 \%$ le VIH.

Le Ministère de la Santé du Burkina Faso a récemment initié une importante réorganisation des services de soins de santé du pays. Parmi les questions en considération figurent l'élaboration d'un programme capable de satisfaire la demande de planification familiale dans la population essentiellement rurale à travers une stratégie rentable et aussi l'intégration d'autres services de santé en matière de la reproduction dans le programme conventionnel de santé maternelle et infantile et de planification familiale. Pour tester une telle stratégie, un projet innovateur à base communautaire est en train d'être mis en œuvre dans la province du Bazèga. Le site du projet est une province rurale de $5.599 \mathrm{~km}^{2}$ située approximativement à 40 kilomètres au sud de Ouagadougou, la capitale du Burkina Faso. La province compte 10 départements, deux districts, 388 villages et une population de 388.562 habitants selon le recensement de septembre 1994. Chacun de ces districts dispose d'un centre médical de référence bien équipé et il y a 38 formations sanitaires à travers toute la province.

La base de l'intervention est la formation, le soutien et la supervision d'agents communautaires volontaires de santé de la reproduction, l'amélioration des formations sanitaires par le recyclage du

\footnotetext{
5 Olukoya, Adepeju, Christopher Elias. « Perceptions of reproductive tract morbidity among Nigerian women and men ». Reproductive Health Matters. 7 : 56-65 ; May 1996.

${ }^{6}$ Zuryak, H.H Khattab, N. Younis, O. Kamal and M. El-Helw. « Combining women's reports with medical diagnosis of reproductive morbidity condition in Egypt». Studies in Family Planning. 26(1) : 14-21; 1995.

${ }^{7}$ Meda, et al. 1995. Bobo-Dioulasso, Burkina Faso.
} 
personnel de santé et l'équipement minimum standard des formations sanitaires. Les agents communautaires ont la responsabilité d'organiser des activités d'éducation en matière de planification familiale, de MGF, de IST/VIH, paludisme et diarrhée; ils fournissent aussi des méthodes contraceptives (pilule, spermicide, condom) de la chloroquine et de SRO. Les activités éducationnelles en matière de IST/VIH abordent les aspects suivants :, les différents types de IST, leurs signes, symptômes et conséquences et les façons de prévenir et de gérer les IST/VIH.

Cette étude a exploré les perceptions des hommes et des femmes de la morbidité du tractus génital dans le Bazèga, avec un accent particulier sur les symptômes et les conséquences et sur l'impact de ces expériences sur le comportement de recherche d'assistance et l'auto médication. La compréhension de l'expérience des femmes et des hommes de ces problèmes aidera plus tard les agents chargés des prestations de services dans la conception de matériels d'IEC appropriés et l'élaboration des approches éducationnelles des questions et guidera à l'adéquation des formations en matière de prise en charge des IST à l'intention des prestataires communautaires (non professionnels) et cliniques (professionnels).

\section{Objectifs de l'étude}

L'objectif final est de contribuer à l'élaboration d'interventions communautaires et cliniques appropriées dans la prise en charge des IST/VIH au Bazèga à travers une meilleure compréhension des perceptions, définitions et descriptions des morbidités de la reproduction par les hommes, les femmes et les prestataires de services.

Les objectifs immédiats sont la collecte de l'information sur :

- le vocabulaire utilisé pour décrire les morbidités de la reproduction ;

- la connaissance des différentes infections du tractus génital;

- les expériences des femmes et des hommes sur les morbidités de la reproduction et leur comportement de recherche de la santé qui en découle ;

- les perceptions que les hommes et les femmes ont des causes, symptômes, conséquences, stratégies de prévention et thérapies des morbidités de la reproduction ;

- l'impact de la morbidité du tractus génital sur les interactions conjugales ;

- les perceptions des agents de santé et des agents de services à base communautaires de la prise en charge des morbidités de la reproduction.

\section{Définitions des concepts}

- Morbidité de la reproduction :

maladies liées à la reproduction, en particulier les infections du tractus de la reproduction.

- Infections du tractus génital: infections des organes reproducteurs de la femme et chez l'homme. Elles peuvent être symptomatiques lorsqu'elles sont accompagnées de douleurs pelviennes, ou asymptomatiques.

- Infections sexuellement toutes les infections virales ou microbiennes transmises lors transmissibles : des relations sexuelles.

- Interactions conjugales : communication entre épouses et époux portant sur des questions de santé en matière de la reproduction. 


\section{Approche méthodologique}

Cette étude a utilisé quatre différentes approches méthodologies de recherche qualitative pour collecter et analyser ces informations : le listage libre, les groupes de discussions dirigées (GDD), les entretiens individuels approfondis (EIA) et le tri par piles. Toutes les données ont été recueillies au niveau de la communauté en langue locale, le Mooré. Le Français a été employé pour certains entretiens avec les prestataires.

Avant la collecte des données sur le terrain, l'équipe de recherche, composée de deux enquêtrices et d'un enquêteur (ayant tous une formation de base en sciences sociales), a reçu une formation de cinq jours en collecte de données qualitatives et sur les questions à discuter. Le concours d'un traducteur de l'Institut National d'Alphabétisation (INA) a permis de bien assimiler les guides en Mooré. Les représentants de la DSF, le coordonnateur et le Population Council ont procuré ladite formation. Un pré-test des guides de collecte des données a été effectué afin de mieux adapter les questions au contexte et d'harmoniser la compréhension par rapport au public cible.

La collecte des données s'est effectuée autour de l'aire de couverture de onze formations sanitaires de la province du Bazèga. L'équipe de recherche a mis l'accent sur la sélection d'hommes, de femmes et de prestataires de service issus d'une gamme variée de groupes ethniques et socio-économiques de la zone du LSC . La sélection des échantillons s'est faite sur la base d'un choix au hasard d'hommes et de femmes.

\begin{tabular}{|c|c|c|c|c|c|c|}
\hline Tableau 1. & \multicolumn{6}{|c|}{ L'échantillonnage par groupe cible et technique utilisée } \\
\hline $\begin{array}{l}\text { Population } \\
\text { étudiée }\end{array}$ & $\begin{array}{l}\text { Groupes des di } \\
\text { par groupe }\end{array}$ & cussions & & Free listing & EIA & Tri par piles \\
\hline Femmes & $\begin{array}{l}\text { Célibataires < } \\
25 \text { ans } \\
\text { (2 groupes) }\end{array}$ & $\begin{array}{|lll|}\text { Mariées } & 25 & \text { à } \\
40 \text { ans } & & \\
\text { (2 groupes) } & \\
\end{array}$ & \begin{tabular}{|l|} 
Mariées plus \\
de 40 ans \\
(2 groupes)
\end{tabular} & \begin{tabular}{|l} 
Plus de \\
14 ans \\
(1 groupe)
\end{tabular} & $\begin{array}{l}\text { Plus de } 14 \text { ans } \\
\text { (20 individus) }\end{array}$ & $\begin{array}{l}\text { Plus de } \\
14 \text { ans } \\
\text { (1 groupe) }\end{array}$ \\
\hline Hommes & $\begin{array}{l}\text { Célibataires < } \\
25 \text { ans } \\
(2 \text { groupes })\end{array}$ & $\begin{array}{l}\text { Mariés } 25 \text { à } 40 \\
\text { ans } \\
(2 \text { groupes })\end{array}$ & & & $\begin{array}{l}\text { Plus de } 14 \text { ans } \\
\text { (20 individus) }\end{array}$ & $\begin{array}{l}\text { Plus de } \\
14 \text { ans } \\
\text { (1 groupe) }\end{array}$ \\
\hline $\begin{array}{l}\text { Prestataires } \\
\text { communautaires } \\
\text { Prestataires } \\
\text { cliniques }\end{array}$ & & & & & $\begin{array}{l}10 \text { individus } \\
10 \text { individus }\end{array}$ & \\
\hline
\end{tabular}

A la suite de la sortie de collecte, il a été organisé un atelier de réflexion de quatre jours afin de procéder à l'analyse primaire du contenu des GDD, des entretiens individuels approfondis, du tri par pile et du listage libre. Cet atelier a regroupé l'équipe de recherche, des représentants de la DSF et de Population Council. Il s'agissait d'impliquer les enquêteurs dans le processus d'analyse afin de permettre une meilleure interprétation des données.

Pour la présentation des résultats au niveau de la communauté, nous optons pour celle mettant en exergue les objectifs de l'étude que sont les symptômes ou maladies, la prise en charge et le traitement, l'itinéraire thérapeutique et la prévention, la communication et les interactions conjugales en matière d'infections sexuellement transmissibles. 


\section{RESULTATS}

\section{Au niveau de la communauté}

\subsection{Les infections sexuellement transmissibles : Maladies et symptômes}

\section{Les symptômes des infections et maladies du tractus de la reproduction}

Le listage libre a permis d'identifier le vocabulaire utilisé pour désigner les morbidités et les symptômes relatifs aux organes génitaux et à la région pelvienne. L'encadré ci-dessous indique les principales infections recensées. Cette liste n'est pas exhaustive ; la suite peut être consultée en Annexe A. Les symptômes sont ceux décrits par la communauté. Pour la syphilis, les symptômes mentionnés correspondent à ceux de la syphilis secondaire, phase évolutive de cette maladie. Ce vocabulaire pourrait aider à lever la barrière de communication entre les prestataires et les clients atteints d'Infection Génito-Pelvienne (IGP).

\begin{tabular}{|c|c|c|}
\hline $\begin{array}{l}\text { Terme en langue } \\
\text { nationale, mooré }\end{array}$ & $\begin{array}{l}\text { Terme en } \\
\text { langue française }\end{array}$ & Symptômes \\
\hline Sompissi ou Vilga & Gonococcie & $\begin{array}{l}\text { - Douleur mictionnelle } \\
\text { - Ecoulement de pus à la fin des urines } \\
\text { - Parfois pus épais sortant comme un ver et dans ce } \\
\text { cas, on l'appelle Vilga }\end{array}$ \\
\hline Silgré ou kodogo & $\begin{array}{l}\text { Règles } \\
\text { douloureuses }\end{array}$ & $\begin{array}{l}\text { - Règles abondantes et douloureuses } \\
\text { - Douleurs généralisées } \\
\text { - } \quad \text { Règles pouvant couler pendant un mois } \\
\end{array}$ \\
\hline $\begin{array}{l}\text { Kopelga ou Rengo } \\
\text { sen Yittaore }\end{array}$ & Pertes blanches & $\begin{array}{l}\text { - L'écoulement d'un liquide blanc du sexe de la femme } \\
\text { - Cet écoulement devient rouge au moment des règles } \\
\text { - Change de couleur ou d'odeur en fonction de la } \\
\text { maladie }\end{array}$ \\
\hline Tuma & Syphilis & $\begin{array}{l}\text { - Plaie sur le sexe } \\
\text { - Prurit généralisé } \\
\text { - Plaie sur tout le corps } \\
\text { - } \quad \text { Tue comme le SIDA } \\
\end{array}$ \\
\hline $\begin{array}{l}\text { SIDA ou Banwinga } \\
\text { ou Yiwangui ou } \\
\text { Weegbanga ou } \\
\text { Nongonbanga ou } \\
\text { Kankeega }\end{array}$ & SIDA & $\begin{array}{l}\text { - } \text { Diarrhée chronique } \\
\text { - } \text { Amaigrissement } \\
\text { - Toux } \\
\text { - Blanchiment du corps } \\
\text { - } \quad \text { Fièvre } \\
\end{array}$ \\
\hline Tigengenga & Chancre mou & $\begin{array}{l}\text { - Plaie profonde sur le sexe } \\
\text { - Peut entraîner la détérioration du sexe si aucun } \\
\text { traitement n'est appliqué } \\
\text { - Douleur au sexe }\end{array}$ \\
\hline
\end{tabular}


Bon nombre de morbidités relatives aux organes génitaux et au pelvis ont été mentionnées par les participants aux GDD et aux EIA. Est-ce lié à la proximité du Bazèga avec Ouagadougou et à l'effet de la sensibilisation par les mass média et des agents de services à base communautaire? Notamment la gonococcie, la syphilis et le SIDA sont les plus connues. Dans un passé récent, les IST appelées "Pug-Bansé », maladies des femmes ne le sont plus contrairement à l'étude du Ghana où ce terme est courant $^{8}$; en effet, le vocabulaire local utilisé pour indiquer celles-ci est «taor bansé » ou "maladie de devant». Ces constatations sont aussi bien valables pour les hommes que les femmes. Est-ce lié au fait que c'est le vocabulaire utilisé dans les campagnes nationales de sensibilisation ou à une disposition particulière de prise de conscience positive de la population du Bazèga ?

\begin{tabular}{|c|c|}
\hline 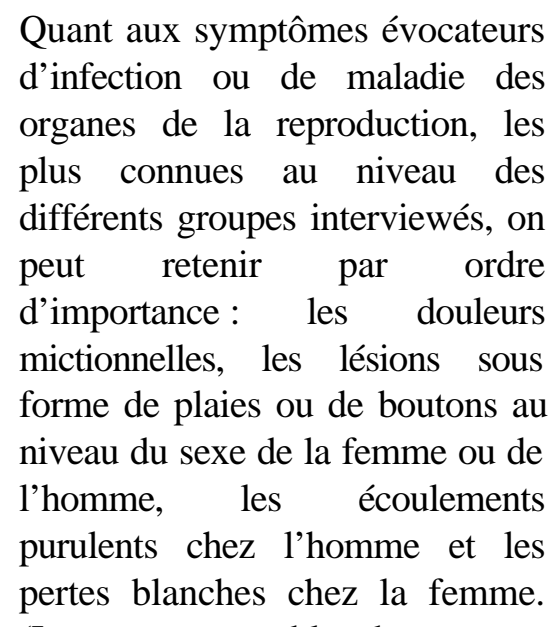 & $\begin{array}{l}\text { Symptômes évoquant des IST mentionnées par les } \\
\text { interviewés } \\
\text { - } \quad \text { Douleurs mictionnelles. } \\
\text { - } \quad \text { Démangeaisons généralisées ou localisées aux parties génitales. } \\
\text { - } \quad \text { Bertes vaginales sentant mauvais, de couleur blanchâtre ou jaunâtre. } \\
\text { - } \quad \text { Gonflement des testicules ou du sexe de la femme. } \\
\text { - Ecoulement purulent ressemblant à un ver ou pus franchement blanc. } \\
\text { - Apparition de boutons prenant la forme d'une crête de coq. } \\
\text { - Règles douloureuses avec ou sans pus. } \\
\text { - } \quad \text { Pucesission de sang à la fin des urines. } \\
\text { Karinsé »). }\end{array}$ \\
\hline
\end{tabular}
(Les pertes blanches sont pathologiques quand elles prennent la couleur purulente ou jaunâtre ou alors quand elles ont une odeur malodorante). Les règles douloureuses et les douleurs au bas ventre de même que des mictions sanguinolentes sont reconnues comme pouvant être des symptômes évoquant une infection du tractus de la reproduction.

La connaissance des symptômes est variable en fonction du groupe et de la tranche d'âge et du sexe. En effet, les hommes de 25 à 40 ans sont ceux qui connaissent le mieux les infections du tractus de la reproduction, plus particulièrement les IST ; les jeunes filles et les garçons de façon moindre. Ces constatations indiquent qu'il faut une attention particulière à l'endroit des jeunes dans les approches futures qui seront développées.

Pour le cas particulier du SIDA, tous les groupes connaissent les symptômes majeurs de présomption de la maladie. Cependant la fièvre prolongée n'est que rarement citée comme symptôme de présomption de l'infection VIH. Il y a également des aberrations relevées par l'ensemble des groupes, "l'envie de bonne nourriture et surtout de la viande» est souvent mentionnée comme un signe évocateur de SIDA.

\section{Les causes des infections du pelvis et des organes génitaux}

Les causes sont variées et multiples selon les enquêtés. En effet on peut distinguer successivement les principales causes suivantes : i) les IPG/IST supposées ou réelles ; ii) les causes traditionnelles et; iii) les causes inconnues ou fatalistes.

\footnotetext{
${ }^{8}$. Navrongo Health Research Center, Perception of Reproductive Health Tract Infection amoung Kassena-Nankana Men and Women in Rural Nothern Ghana ; Symptoms recognition, Perceived Causes, Treatment Choices and Partners Notification, Preliminary report.
} 
De façon générale, il ressort des GDD et des EIA, que seulement la gonococcie ou "chaude Pisse » Maladies pouvant relever du mauvais sort ou «Sompiissi » et le SIDA ont pour cause les • SIDA relations sexuelles. Ensuite le «Touma» ou syphilis est une IST selon les groupes de femmes de 25 ans et plus et chez les groupes d'hommes (moins de 25 ans et de 25 à 40 ans). En dehors des femmes de plus de 40 ans et des hommes de plus de 25 ans qui précisent la notion d'agent causal (microbes ou «banbissi »), les autres groupes n'en font pas cas. Les écoulements urétraux sont reconnus comme des IST chez les groupes de femmes de plus de 25 ans à 39 ans et chez celles de plus de 40 ans.

\author{
- Gonococcie \\ - Syphilis \\ - Poux du pubis \\ - Démangeaisons \\ - Règles abondantes \\ - Chancre mou \\ - Pertes vaginales \\ Maladies pouvant relever de mauvais génies \\ - Syphilis mais seulement en plein soleil \\ (soleil au zénith)
}

Cependant certains participants, surtout les jeunes, estiment que l'on contracte la bilharziose et la Maladie pouvant être liée à une violation d'interdits - SIDA gonococcie en pissant au même endroit qu'une personne déjà atteinte de la maladie. Pour le cas spécifique du SIDA, les mouches, les moustiques et le port de vêtement d'un sidéen et la mauvaise alimentation, la salive peuvent être sources de contamination. Aussi des maladies comme la hernie est reconnue comme une IST selon les femmes de plus de 25 ans.

\title{
Les causes traditionnelles
}

En Afrique, l'on a souvent coutume de dire qu'une maladie n'est jamais gratuite. C'est souvent l'effet de mauvais sort ou de génie ou de transgression des interdits. Dans cette étude, les causes traditionnelles se résument au «mauvais sort », à la violation d'interdit, aux faits de mauvais génies et enfin au piège ou "bindego ». Le SIDA est rarement mentionné comme un mauvais sort mais plutôt comme une maladie relevant d'une violation d'interdits qu'aucun participant n'est arrivé à définir ; par contre le «koodgo » ou règles abondantes, les morpions ou «pend karinsé », la gonococcie et la syphilis ou «touma» sont souvent mentionnées comme des maladies relevant du «mauvais sort». Ces affirmations sont surtout constatées chez les femmes de plus de 25 ans, les hommes, que ce soit les jeunes de moins de 25 ans que ceux de plus de 25 ans.

\section{Les causes fatalistes ou inconnues}

Malgré les campagnes de sensibilisation, la perception des causes des infections du tractus de la reproductions sont moins bien connues chez le groupe de jeunes filles et jeunes garçons. La plupart des symptômes ou maladies du pelvis et des organes génitaux sont de causes inconnues dans ces deux groupes. Par exemple, les jeunes garçons prétendent qu'en urinant au même endroit qu'une personne atteinte, l'on peut attraper la maladie. Les femmes de 25 à 39 ans précisent que c'est le simple fait d'inhaler les gaz des urines infectées qui est la source de - "Le sompissii ou chaude pisse peut être dû̀ à un mauvais sort si par un exemple un homme fait la cour à la femme d'un autre » (femme de plus de 40 ans)

- "Les génies peuvent être à l'origine du tuma (syphilis) car on dit que si tu sors sous le soleil brûlant, tu risques de rencontrer les mauvais génies qui vont te donner la syphilis» (femme de plus de 40 ans)

- "On peut attraper la chaude pisse en urinant au même endroit qu'un sujet déjà infecté »

(homme de moins de 25 ans)

- «La syphilis est une maladie due aux rapports sexuels avec une personne infectée, c'est une maladie que Dieu a créée » (homme de plus de 40 ans) 
Les règles abondantes, les règles douloureuses et le SIDA sont des maladies de Dieu. Les pertes blanches, les règles douloureuses et les écoulements urétraux sont de causes inconnues surtout chez les jeunes filles et chez les jeunes hommes de moins de 25 ans. Le condylome est également de cause inconnue chez les groupes de femmes de plus de 25 ans ainsi que chez les jeunes hommes de moins de 25 ans. Les résultats ci-dessus expriment de nouveau qu'il faut accorder une attention particulière aux jeunes générations dans le cadre de la lutte contre les infections génitales et pelviennes. Jusqu'à présent il n'y a effectivement pas de programme destiné spécifiquement aux jeunes surtout en zone rurale.

\section{Conséquences des infections ou maladies du tractus de la reproduction}

Les infections pelviennes et des organes génitaux ont bien sûr des conséquences à court, moyen ou long terme. Les interviewés, hommes et femmes, ont été invités à donner leur perception des conséquences des infections du tractus de la reproduction non traitées. De façon générale, c'est la mort, la contagion de l'entourage, la stérilité, la marginalisation, la destruction des organes et l'abandon par les amis qui sont les éléments les plus mentionnés. Les conséquences sont surtout exprimées par les jeunes garçons.

La perception de la contagiosité et l'expansion des IST se traduit chez les jeunes à travers la citation suivante : "Si quatre personnes sont malades et ne se soignent pas et ils ont des rapports sexuels avec quatre autres personnes, il y aura huit malades et ainsi de suite, le nombre va augmenter» (groupe des hommes de moins de 25 ans) ; toujours chez les jeunes, l'appréciation des conséquences sociales s'exprime également dans la citation suivante : "Si une IST n'est pas soignée, c'est la mort du malade et de ceux qu'il aurait contaminé... Toute personne a son importance dans une famille et la mort du malade va créer un vide au sein du ménage et va effacer tout espoir que les parents avaient en eux à l'avenir » ou encore "une ITR peut ronger le sexe au point de le faire disparaître »(jeunes hommes de moins de 25 ans). Ainsi l'on note des conséquences médicales et sociales.

De plus les interviewés reconnaissent que les ITR sont contagieuses mais parlent plutôt de l'entourage que du partenaire, dans la mesure où des raisons sociales et traditionnelles à la maladies sont souvent évoquées et la contamination sexuelle est souvent occultée. L'analyse selon les GDD, les EIA et les tranches d'âge ne montre pas de différence dans les conséquences répertoriées.

Conséquences des ITR non traitées évoquées par ordre d'importance

- La mort

- La stérilité

- La contamination de l'entourage

- La marginalisation du malade par ses amis

- L'aggravation de la maladie

- L'amaigrissement

- La tristesse de l'entourage et du malade

- L'impossibilité d'avoir des rapports sexuels La difficulté d'avoir une femme ou un mari

\subsection{La prise en charge et le traitement des personnes malades par la communauté}

La dynamique de prise en charge des malades atteints d'IST au niveau communautaire est un phénomène nouveau qui mérite encore une exploration approfondie par les acteurs intéressés par les services à base communautaire. Ainsi une des préoccupations de cette étude a été de savoir ce que la communauté peut faire en terme de prise en charge des malades d'IST. Pour la majorité des interviewés, il faut amener les malades à la formation sanitaire, les aider dans la recherche de traitement adéquat. Le counseling n'est pas du reste dans la prise en charge des malades ; en effet, tous les groupes et interviewées proposent d' " encourager les malades afin qu'ils aient foi en la guérison » et certains de satisfaire aux besoins des malades en les aidant à cultiver, à faire la lessive ou la cuisine.

Pour une maladie incurable comme le cas du SIDA, la proposition ultime est de « satisfaire à tous les besoins des malades et s'occuper d'eux jusqu'à leur dernier jour» ou encore selon les jeunes filles de moins de 25 ans, " de cajoler le malade pour qu'il ne souffre pas de la maladie» ou de " soigner le malade même si on sait qu'il va en mourir». Les jeunes filles et les jeunes garçons sont ceux qui ont une approche réconciliante et compatissante dans la prise en charge : "pour quelqu'un 
qui est malade d'une ITR, on peut l'amener au dispensaire d'abord ; s'il ne trouve pas la santé, on l'amène chez le guérisseur et s'il ne trouve toujours pas la santé, on arrête tout rapport sexuel avec lui afin de ne pas se faire contaminer et pour pouvoir le soutenir car tout malade a besoin d'un soutien; si on se fait contaminer qui va soutenir qui ?... Pour les malades de SIDA, il ne faut pas les fuir car on a entendu dire que la maladie ne se transmet pas par l'eau... Il faut aider le malade jusqu'à sa mort, l'aider à nettoyer sa maison, le soulever pour lui donner à manger et chercher à le soigner même si on sait que le remède n'existe pas » (jeunes hommes de moins de 25 ans). Le groupe des hommes de plus de 40 ans suggère l'isolement des malades atteints de SIDA pour qu'ils ne contaminent pas d'autres personnes.

Des attitudes fatalistes sont parfois aussi notées çà et là au cours des GDD et des EIA, c'est celles de prier pour les malades. Des propos sexistes ont été exprimés par des hommes de plus de 25 ans qui suggèrent la répudiation de la femme si celle-ci contracte une IST. Les hommes ont tendance à penser qu'une femme qui a une IST a sans doute trompé son mari ou son partenaire.

\subsection{L'itinéraire thérapeutique}

Concernant ce volet, jeunes comme personnes âgées, mariés comme non mariés sont unanimes à dire que les personnes malades d'IGP vont se soigner soit au dispensaire, soit chez le guérisseur ou chez toute autre personne capable de les aider. Mais les jeunes filles ont surtout fait ressortir l'idée d'une prière à l'église (chez le pasteur) qui selon elles, est très efficace. Pour ce qui est du traitement au dispensaire, l'initiative de la prescription revient à l'agent de santé selon le type de IST que celui-ci a diagnostiqué. Des enquêtés ajoutent que si son traitement s'avère inefficace, les malades se rendent chez le guérisseur ou chez le marabout : "Comme la tradition n'est jamais finie, on peut dire que c'est un mauvais sort qu'on lui a jeté ». Il peut arriver aussi que le malade fasse d'autres traitements avant d'aller au dispensaire.

\section{Traitement des IST selon les enquêtés}

- Dans le traitement des prurits, on utilise des racines de plantes les noms n'ont pas été cités car " on ne divulgue pas de telles connaissances».

- Pour ce qui est du traitement du «raboukienka», il faut d'abord trouver un homme, ensuite utiliser un produit qu'on applique sur le sexe de l'homme ou de la femme et on les invite à entretenir des rapports sexuels ; après cela il doit y avoir ouverture du canal vaginal de la femme qui était bouché. Mais un tel homme n'aura plus longue vie. Cependant, on dit qu'il existe des hommes qui sont des spécialistes en la matière.

- Pour le traitement du «goundi », il faut faire brûler le «goundi» (nom des mille pattes attribué à la maladie en Mooré), l'écraser et appliquer la poudre sur la partie infectée.

- Pour le traitement du "tuma», on utilisait un épis de maïs rongé par les termites pour frotter le sexe afin de l'en débarrasser des boutons et du pus. Ce procédé laissait apparaître une plaie que l'on soignait après.

- Dans le cas du «roudsenéga», on écrasait des fourmis dans la farine de mil et on en faisait manger à l'enfant ou bien encore, on utilisait des racines de "boulyoanka » (plante qui pousse en saison hivernale) ensuite on les prépare comme du couscous et on le donne à manger à l'enfant. On peut aussi faire boire du vinaigre ou du jus de tamarin pour calmer la douleur.

- Dans le cas du «zig voaka» ou crête de coq, il faut procéder à une nouvelle ablation de ce qui est supposé être un clitoris.

- Pour les maladies telles que les «pen karensé » ou morpions dont les causes sont liées à un sort jeté, il faut rechercher les soins auprès des personnes qui sont à l'origine du sort. 
Le traitement traditionnel a été unanimement reconnu dans ses forces et ses limites. C'est ainsi que certains hommes pensent que le traitement traditionnel n'est plus efficace de nos jours "parce que le monde a changé ». Le traitement traditionnel est de plus fréquent recours ; pour chaque maladie, un traitement traditionnel est souvent tenté. C'est la raison pour laquelle les malades arrivent secondairement dans les formations sanitaires à un état assez avancé de la maladie. Cet état de fait joue énormément sur les aspects de prise en charge en terme de coût, de durée du traitement et des conséquences à court ou long terme.

\section{L'automédication}

L'automédication est le fréquent recours dans le traitement des maladies ou symptômes évoquant les IST. Pour le traitement de la gonococcie ou «soumpiisi », on utilise du Tonic auquel il est ajouté du «toupaye $e^{9}$ et que l'on boit. Ou encore du «koutoukou» (liqueur SOPAL) en plus du « NESCAFE » et en plus du «toupaye ». Ou encore, on fait bouillir des écorces d'un arbre (dont le nom n'a pas été communiqué), on y ajoute du "toupaye » pour boire. Pour le traitement des règles douloureuses, les malades prennent des médicaments appelés "wobgo », "éléphant» ou " 14 », comprimé sur lequel est inscrit la lettre E. Cela calmera les douleurs. Dans le cas des règles abondantes et douloureuses, il suffit de donner de la bouillie sucrée au malade, ainsi le sang contenu dans son ventre est évacué et le malade recouvre la santé au bout de 3 jours.

En résumé, les jeunes pensent que le traitement médical est meilleur au traitement traditionnel «parce que dans le traitement traditionnel la guérison est une question de chance ». Mais d'une manière générale les individus interrogés disent que le traitement, qu'il soit médical, traditionnel ou spirituel, "le meilleur est celui qui guérit», ce qui témoigne de l'ouverture de la population par rapport au traitement moderne si celui-ci est disponible et de qualité. C'est un atout à mettre à profit pour les interventions futures.

\subsection{La prévention des IGP/IST}

Les IGP/IST ont été reconnues comme des maladies graves et mortelles par les enquêtés. Et pour s'en prémunir, la majorité propose l'utilisation de la capote lors des rapports sexuels, la fidélité, suivi des conseils des agents de santé, et le traitement des partenaires : "Ce sont les gens qui cherchent la maladie, la maladie elle ne cherche personne ; en utilisant la capote, on protège sa vie mais aussi on se prive d'enfants » (jeunes de moins de 25 ans). Mais quelques individus disent qu'il faut se donner des conseils mutuellement. Car tout le monde ne connaît pas les IST et leurs mesures préventives. En exemple, ils disent qu'il faut éviter d'utiliser des objets souillés (lames, seringue, pratique de l'excision ....). Toujours en ce qui concerne le SIDA et les autres IST, il faut s'abstenir d'avoir des rapports sexuels avec des personnes malades.

L'ignorance persiste également au sein de la population car celle-ci ne connaissant pas toujours l'origine des maladies se demande comment les prévenir ; c'est à la fois là une attitude fataliste et une ignorance qui sont à l'origine de certains comportements à risque comme le souligne ces deux citations : "Il faut oublier les maladies, car si on les oublie, elles aussi vont nous oublier » ; "Si on ignore d'où vient la maladie comment peut-on s'en prémunir?»

\footnotetext{
${ }^{9}$ Le « toupaye » est de la tétracycline vendue de façon illicite sur la place du marché que le sens commun utilise pour le traitement des symptômes de IST.
} 


\subsection{La communication et les interactions conjugales en matière d'IGP/IST}

\section{La communication en matière d'IGP/IST}

En ce qui concerne la communication, la tendance générale qui se dégage des GDD et EIA est que les gens parlent peu entre eux au sujet des maladies liées aux organes de la reproduction. Si le cas se produisait cette communication a lieu entre femme/femme, entre homme/homme ou entre amis. Pour ce qui est des jeunes filles la majorité se confie à leur mère ou à leur tante. Les parties génitales sont les parties intimes et de ce fait devraient être gardées cachées ; pour toutes ces raisons, les pathologies liées aux organes de la reproductions sont perçues comme des maladies honteuses et des maladies liées à un comportement déviant, c'est à dire proscrit par les normes sociales ; ce qui constitue une contrainte énorme en matière de communication sur les infections génitales.

Une autre idée non moins importante est que les personnes atteintes d'IST préfèrent se confier à des gens qui peuvent avoir des remèdes à leur mal (agents de santé, guérisseurs, pasteurs, accoucheuses villageoises). Au regard de toutes ces idées, il ressort une certaine honte et surtout la discrétion à parler avec son entourage en matière de IST/SIDA car disent-ils, "la nouvelle va loin si un étranger l'apprend». Cette citation exprime le fait que l'on ne souhaiterait pas qu'une autre personne apprenne que l'on porte une IST ; elle exprime aussi une certaine honte car les IST sont synonymes de vagabondage sexuel.

L'élément important qui est également souligné est que pour la communauté si on parle peu des IST, c'est que : "on est préoccupé par d'autres problèmes tel que la famine », car "quelqu'un qui a faim est un fou ». La communauté ne perçoit pas les conséquences des IST et donc ne les considère pas comme une priorité. Peut-être que la prise en charge des IST devrait se faire dans un cadre global d'intégration des services.

\section{Les interactions conjugales en matière d'IGP/IST}

Comment les couples devraient-elles s'y prendre pour annoncer une infection pelvienne diagnostiquée ? La majorité des femmes enquêtées trouvent qu'il est plus facile pour le mari de parler à la femme d'une IST dont il souffre sans être inquiété. Par contre, il y a une difficulté pour la femme, si c'est chez la femme que la maladie a été diagnostiquée en premier lieu, d'en parler au mari. Ces difficultés se situent à deux niveaux :

- la femme doit obtenir l'autorisation de son mari avant d'aller demander des soins.

- les infections sexuellement transmises sont synonyme d'infidélité et par conséquent, la femme est accusée d'infidélité et peut même être répudiée.

Si cette situation fait que les échanges au sein des couples sont très limités, de façon spécifique, la majorité souhaite que les femmes et les hommes fassent l'effort de comprendre car "la maladie n'est pas une marchandise que l'on voit et qu'on achète, la femme n'a donc pas acheté la maladie ».

Pour le traitement des partenaires, la majorité des personnes interviewées dit que «l'homme explique calmement la situation à la femme et l'invite à aller se faire soigner au dispensaire ». Mais quand il s'agit de la femme, il est préférable que celle-ci demande l'intervention du prestataire clinique auprès de son mari car cela peut atténuer les disputes au sein du couple. 


\section{RESULTATS CLES AU NIVEAU DE LA COMMUNAUTE}

\section{IST : Maladies et symptômes}

- Il existe des termes et expressions spécifiques locales pour désigner les IST dans les deux zones d'étude. Les symptômes les plus courants sont : les douleurs mictionnelles, les lésions et plaies, les boutons au niveau du sexe. Leurs causes peuvent être traditionnelles, supposées ou réelles ou inconnues.

- La connaissance de ces infections varie selon la tranche d'âge et le sexe. Par contre tous les groupes d'étude connaissent les principaux symptômes du VIH.

- Les IST non traitées provoquent la mort, la stérilité, la marginalisation et la destruction des organes génitaux.

\section{La prise en charge et le traitement des malades par la communauté}

- Des attitudes fatalistes ou sexistes sont souvent évoquées. Cependant, accompagner le malade à la formation sanitaire ou l'aider à trouver un traitement adéquat sont les priorités de la communauté.

\section{Itinéraire thérapeutique et prévention}

- Le prestataire, le guérisseur traditionnel, la prière et l'automédication sont les recours en cas d'IST.

- Le traitement traditionnel est perçu comme étant de moins en moins efficace. Cependant, le meilleur traitement est celui qui apporte la guérison.

- La capote, la fidélité, les conseils des prestataires et le traitement des partenaires sont les moyens de prévention les plus cités.

\section{Communication et inter action conjugale en matière d'IST}

- Par gêne ou par honte, les gens parlent peu entre eux au sujet des IST. Les discussions ont lieu entre hommes, entre femmes, entre amis, de fille à mère ou à tante.

- Si l'homme a plus de facilité à parler d'une IST dont il souffre, la femme quant à elle doit obtenir l'autorisation de son mari (prise en charge financière des soins) et demander l'intervention du prestataire. 


\section{Au niveau des prestataires professionnels et communautaires}

En rappel, l'objectif était d'étudier les perceptions des agents de santé et des agents de services à base communautaire de la prise en charge des morbidités de la reproduction. En plus donc de la collecte des données auprès de la communauté, la présente étude s'est intéressée aux prestataires. On y distingue d'un côté les prestataires professionnels (personnel clinique) des formations sanitaires et les prestataires non professionnels. Il s'agit dans ce dernier cas des agents de santé communautaire et des agents DBC.

Le volet étude au niveau des prestataires requiert deux niveaux d'intervention (professionnels et non professionnels) à tel point que les éléments suivants ont été explorés pour chacun des deux groupes de prestataires. Pour la présentation des résultats au niveau des prestataires, nous optons pour celle mettant en exergue les objectifs de l'étude que sont: l'ampleur des pathologies génitales, les conséquences des infections génitales, le traitement et la prévention, le type de communication entre prestataires et clients, enfin les propositions faites en vue de l'amélioration de la prise en charge des IGP/IST.

\subsection{Population et ampleur des pathologies génitales}

Infections sexuellement transmissibles fréquemment rencontrées dans les

Selon les prestataires de services de santé professionnel et non professionnel, la tranche de la population la plus touchée par les IST est celle comprise entre 15 et 49 ans, l'âge de reproduction sexuellement active tant chez les hommes que chez les femmes. Les hommes sont les plus touchés, surtout la tranche jeune qui a une plus grande activité sexuelle. Cet état s'explique par le fait que les hommes se présentent plus facilement en consultation pour les affections du tractus uro-génital. Parmi ces hommes les plus touchés on remarque surtout ceux qui reviennent de la Côte d'Ivoire et les commerçants. Les migrants et les personnes ambulantes constituent les personnes à risque. Les femmes en âge de procréer et particulièrement les femmes mariées sont aussi touchées par les IST. Les femmes se présentant zones de couverture de FS selon les prestataires professionnels et non professionnels

- Les vaginites

- Les leucorrhées sont liées aux IST

(comme la gonococcie)

- Les prurits vulvaires sont liées aux IST

- Blennorragie ou gonococcie ou soumpissi

- La syphilis

- Les candidoses

- Les dysménorrhées

- La stérilité

- Le chancre mou

- La crête de coq ou condylome

- La bilharziose

- $\quad$ Le SIDA dans les formations sanitaires pour une consultation prénatale souffrent d'IST telles les vaginites, les candidoses, les prurits vulvaires et les leucorrhées.

Il est important de noter que les professionnels ne mentionnent pas les trichomonoses vaginales dont le diagnostic est aisé par l'odeur caractéristique des pertes qui l'accompagnent. Aussi, seulement quelques prestataires non professionnels ont mentionné que la syphilis est une maladie qui n'existe plus, en précisant, depuis l'année 1946.

\subsection{Problèmes et conséquences des IGP/IST non traitées}

Retenons qu'aucun prestataire clinique ou communautaire ne parle de conséquences psychologiques, dimensions importantes à prendre en compte dans les IST. Certains malades en souffrent énormément au point de vivre les symptômes «psychologiquement». 


\section{a) Conséquences médicales chez les professionnels}

Chez l'homme les conséquences sont la stérilité, le rongement du gland, la perte de virilité, une hémorragie lors des rapports sexuels, des douleurs mictionnelles. Chez la femme, on retiendra les conséquences suivantes : une inflammation du col de l'utérus, puis de l'utérus et enfin des trompes, une septicémie ou infection généralisée, une stérilité, des avortements à répétition, des accouchements prématurés, une hémorragie lors des rapports sexuels, des douleurs mictionnelles et pelviennes, des dispareunies (douleurs lors des rapports sexuels), une contagion du bébé au moment de l'accouchement, une mutilation de l'appareil génital.

\section{b) Conséquences médicales chez les non professionnels}

Les conséquences sont la stérilité, le rongement du gland et les douleurs mictionnelles.

\section{c) Conséquences sociales chez les professionnels}

Si le SIDA entraîne la mort, les autres IST peuvent entraîner la folie, la propagation de la maladie au sein de la communauté, des disputes dans le foyer s'il y a stérilité avec manque d'enfant. Dans ce cas, la femme est souvent indexée. Il y a une gêne face à la communauté due à l'absence d'enfant. Il peut en résulter marginalisation et isolement des malades (refus de partager un repas dans le même plat ou même de lui serrer la main).

\section{d) Conséquences sociales chez les non professionnels}

La mort, la propagation de la maladie et la marginalisation du malade sont les éléments les plus souvent évoqués.

\section{e) Conséquences économiques selon les professionnels}

On retiendra l'épuisement des fonds financiers parce que le malade est handicapé et ne sera plus productif. Il est à la charge des autres membres de la communauté. Appauvrissement dû aux grosses dépenses consacrées aux soins.

\section{f) Conséquences économiques selon les non professionnels}

Les dépenses excessives pour la recherche de la santé et l'endettement sont les principales conséquences évoquées. Le prix du produit lorsqu'il existe au niveau du dépôt pharmaceutique ou de la pharmacie villageoise est souvent au-delà de la portée de la majorité des membres de la communauté.

\subsection{Traitement et prévention}

\section{a) Traitement curatif selon les prestataires professionnels}

Selon les prestataires cliniques toutes infections pelviennes peuvent se guérir (en dehors d'une infection liée au SIDA), si le traitement est bien suivi. De ce fait, devant une infection pelvienne, le premier geste médical est l'administration d'un traitement au patient en veillant à ce que le partenaire soit également soigné. Si ce dernier est réticent, le prestataire lui fait envoyer une convocation ou saisit toute occasion (présence à la formation sanitaire) pour le rencontrer. Conscients de la contagiosité des IST, ils conseillent en ce moment au couple d'éviter tout rapport sexuel au cours du traitement jusqu'à guérison complète. Par la suite, le prestataire procure des conseils aux clients pour qu'ils évitent une récidive de la maladie (diminuer le nombre de partenaires si possible, utiliser le condom, être propre et bien entretenir ses sous-vêtements). Si après un autre examen du client le prestataire constate que l'infection persiste ou si cette infection est très grave, celui-ci le réfère à une structure sanitaire plus compétente pour les soins requis.

\section{b) Traitement curatif selon les prestataires non professionnels}

Les prestataires communautaires perçoivent seulement leur rôle dans le counseling et dans la référence du malade à la formation sanitaire pour les soins curatifs en lui demandant de faire soigner son partenaire. En effet, ils préconisent une discussion en tête à tête (counseling) pour le questionner sur 
son mal. Ensuite, ils l'orientent vers une formation sanitaire et l'y accompagnent s'il le faut (en cas de gêne ou honte) pour expliquer la maladie au prestataire professionnel.

\section{c) Prise en charge des malades de SIDA selon les prestataires professionnels}

Pour ce cas spécifique, l'attitude globale des prestataires cliniques se recoupe sur le traitement des symptômes.

Pour ce qui concerne la demande d'un test sérologique, trois tendances se dégagent; ceux qui estiment qu'il faut faire un test, ceux qui pensent qu'ils n'ont pas le droit de le faire, et ceux qui ne savent quoi faire. La troisième tendance est liée au fait que les prestataires n'ont pas reçu de formation en la matière. Cependant on a trouvé une attitude positive chez les prestataires qui recommandaient l'utilisation des condoms. Pour la prise en charge

Quelques propos recueillis :

- "Si quelqu'un est atteint du SIDA, il n'y a plus de conseils à lui donner. Il est inutile de le conseiller désormais ; cela ne lui servira pas. »

- «Si quelqu'un est malade du SIDA, il est foutu.»

- "Je ne peux pas savoir comme ça que quelqu'un souffre de SIDA. Selon ce qu'on nous a enseigné, je ne le sais que lorsque la personne est dans une phase avancée. Malheureusement en ce moment là, je ne peux plus faire quoique ce soit. »

- "Ceux qui ont connu des IST sont plus exposés au SIDA. Par exemple, pour le cas de la syphilis, il y a des plaies qui peuvent être des voies par lesquelles le virus du SIDA passe. »

des malades, une formation s'impose comme en témoignent les citations contenues dans l'encadré à côté.

\section{d) Prise en charge des malades de SIDA selon les prestataires non professionnels}

Quant aux prestataires communautaires, ils ont une attitude favorable pour la prise en charge des cas de SIDA ; ceci pourrait être utilisé pour la prise en charge au niveau communautaire. En effet, pour la majorité d'entre eux, il faut d'abord préparer le client en allant discuter régulièrement avec lui, ce qui va le mettre en confiance pour l'amener à se confier à propos de son mal et pour le soutenir moralement. On doit lui donner des conseils sur l'utilisation de condoms pour les rapports sexuels et le référer vers une formation sanitaire appropriée pour se faire examiner. Pour ne pas l'effrayer, il ne faut pas dire au client qu'il est atteint du SIDA.

\section{e) Prévention des IST et rôle des prestataires selon les prestataires cliniques}

L'arme la plus efficace contre un problème de santé demeure la prévention. Les prestataires professionnels préconisent l'information, l'éducation et la communication (IEC) à l'intention des clients. Le prestataire se doit d'informer le malade s'il est atteint d'IST et qu'il doit amener ses partenaires pour les soins. Une bonne hygiène sexuelle par l'utilisation du condom peut aussi contribuer à prévenir les IST. Pour soutenir les activités d'IEC auprès de la communauté, les visites à

- $\quad$ "Si tu caches un seul cas de maladie contagieuse, d'ici une année tu fera trois victimes de plus. »

- "La capote vous aide à prendre ce que voulez et puis laisser l'autre partir avec son SIDA. » domicile afin de sensibiliser les familles, les causeries dans le but d'éclairer la population sur les maladies, l'organisation de semaine de sensibilisation sur les IST, sont entre autres des approches proposées pour prévenir l'expansion des IGP surtout celles sexuellement transmissibles.

Toujours selon ces prestataires, la communauté doit prendre ses responsabilités et reconnaître l'existence des maladies et désigner des membres issus de cette communauté pour la sensibilisation. Cette méthode est mieux acceptée par la population. De plus, l'on devrait engager des actions qui aideraient les populations à ne pas se gêner pour aller en consultation afin de permettre un diagnostic précoce et un traitement à temps. Cependant, la manière d'aborder la population est controversée dans le milieu des prestataires comme le souligne le box suivant : 
- "Quand on parle de SIDA la population s'intéresse plus à l'aspect clinique qu'à la prévention et quand tu n'es pas prudent en la matière tu vas parler et ils vont prendre les symptômes afficher sur un individu et ça crée des problèmes. Il faut donc être souple et dire seulement que le mal existe et qu'il est mortel et dire comment se prémunir.»

- «Si on insiste sur les conseils, on arrive toujours à les accepter.»

\section{e) Prévention des IST et rôle des prestataires selon les prestataires communautaires}

En résumé, les prestataires communautaires pensent à une organisation endogène communautaire et l'adoption de comportements sexuels sains pour la prévention des IST. Effectivement, ils recommandent la fidélité ou l'utilisation de la capote même si celle-ci n'est pas un remède, le conseil mutuel et le bon suivi des conseils du corps médical. La communauté doit aussi s'organiser (caisse communautaire par exemple) pour prendre en charge ou venir en aide aux malades démunis. Ainsi, quiconque souffre d'une maladie contagieuse devrait le dire pour qu'on puisse prendre des précautions. Enfin, ceux-ci perçoivent leur rôle dans la prévention au sein de leur communauté, comme l'indique l'encadré ci-dessous. C'est une situation favorable pour la mise en place de stratégies à base communautaire. L'organisation de référence vers les formations sanitaires peut aussi bien être organisée autour des agents à base communautaire.

\subsection{La communication autour des IGP/IST}

L'étude a cherché à savoir si les clients ou les gens de la communauté discutaient facilement et ouvertement des maladies pelviennes et génitales et • les raisons.

\section{a) Communication au sein de la communauté}

Le rôle des prestataires communautaires. Ceux-ci perçoivent leur rôle en terme surtout de prévention en sensibilisant leur communauté et référant les malades.

- Encourager les clients à parler de leurs maladies sans gêne afin d'avoir le traitement requis.

- Profiter des occasions de rassemblement des jeunes (marché, soirées dansantes) pour leur prodiguer des conseils sur le contrôle de la sexualité.

- Conseiller les malades pour qu'ils se soignent avec leurs partenaires pour les IST.

- Accompagner le malade, s'il le faut, à la formation sanitaire.

Selon les prestataires cliniques

En général les malades ne parlent pas facilement et ouvertement des affections touchant aux organes génitaux, sauf parfois en consultation. Le client consulte le prestataire

- Aller dans un village ou dans un quartier où l'on ne vous connaît pas pour espérer réussir la sensibilisation. Les gens ne pouvant pas parler de leurs maladies à ceux qu'ils connaissent, se conféreront plus facilement à vous.

- Sensibiliser la population sur la salubrité et l'hygiène sanitaire, condition nécessaire pour la santé (on évite ainsi les maladies comme le « roudsenega » ou le « soumpissi »).

lorsque l'infection est très avancée avec des complications et surtout quand les douleurs deviennent insupportables. Le constat est que les hommes vont vers les prestataires hommes et les femmes vers les prestataires du même sexe. Les clients viennent consulter et parlent aisément des maladies autres que les IGP/IST.

\section{Selon les prestataires communautaires}

Ils sont souvent un passage obligé pour atteindre les prestataires professionnels. La population considère les IGP comme étant des maladies honteuses et en parle seulement lorsque les crises deviennent très douloureuses et insupportables. Les femmes malades passent souvent par le prestataire non professionnel du même sexe qu'elles pour atteindre le professionnel de la santé. Les hommes de leur côté agissent de même. Lorsque les malades se présentent en consultation, ils ne disent pas tout sur les symptômes de leurs maladies. Le coût des produits pharmaceutiques est très élevé. D'autres se soignent d'abord avec des produits pharmaceutiques prohibés qui se vendent au marché avant de se rendre au dispensaire. Les séances de sensibilisation (IEC) à travers le programme de «soins de santé » facilitent l'échange entre malades et prestataires. Cependant ceux qui ont des relations particulières avec les prestataires discutent aisément avec eux sur les IGP. 


\section{b) Disposition des prestataires à discuter d'IGP avec les clients}

Contrairement à la population qui ne parle pas aisément et ouvertement des IGP, les

prestataires professionnels ou non, prétendent discuter sans difficulté des IGP avec les communautés. Le constat est identique pour les deux catégories de prestataires. Rappelons cependant que l'Analyse Situationnelle avait montré que la plupart des prestataires avaient du mal à évoquer ce type d'infection avec les populations de leur aire de rayonnement.

D’autres problèmes demeurent au niveau des prestataires cliniques et qui limitent leur action :

- C'est celui de la langue d'usage dans la zone. Il existe un réel problème de compréhension de la langue locale et le vocabulaire à utiliser pour désigner certaines choses surtout pour ne pas choquer ses interlocuteurs.

- On parle plus souvent de la prévention des infections que de leurs manifestations. Ceci à cause d'une certaine gêne ou de la non maitrise des termes appropriés.

- Le niveau d'instruction généralement bas dans la population limite leur compréhension et fait que les gens eux-mêmes ne sont pas à l'aise pour parler des IGP. Ce qui fait que les prestataires sont plus à l'aise pour discuter avec les jeunes qui ont été à l'école.

\subsection{Propositions pour une amélioration de la prise en charge des clients}

Les prestataires dans leur ensemble demandent de leur fournir du matériel de travail (matériel de soins sanitaires, de laboratoire, matériel de projection de films éducatifs et d'affiches, quoi il souffre, je ne vois pas médicaments, groupe électrogène, poste TV) et du matériel pour pourquoi j'en aurais honte. Je le apprendre aux femmes à faire de la bouillie aux enfants à fin d'intégrer les services.

\section{a) Prestataires cliniques}

Ils suggèrent le recours à l'utilisation de personnes ressources comme un spécialiste venu de Ouagadougou pour les appuyer dans leurs activités. Ce dernier apparaît comme un «grand », ce qui impressionne les participants et donne de l'importance à la séance de sensibilisation. Tous attendent une formation et recyclage sur les maladies et des nouveaux médicaments. Les prestataires sont disposés à mener des activités dans leur formation dans le cadre global de lutte contre le SIDA et les IST.

\section{b) Prestataires communautaires}

La motivation des agents à base communautaire est au centre des débats quand il s'agit de la mise en place d'une approche à base communautaire. C'est le problème qu'évoquent les prestataires communautaires à la question de savoir ce qu'il faut faire pour améliorer la prise en charge des IGP/IST. Ils attendent un soutien en médicaments et en matériel de travail (ciseaux, pince, moyen de déplacement autre que le vélo...) et une aide financière afin qu'ils puissent satisfaire leurs besoins familiaux. Selon eux, ils sont accaparés par cette activité communautaire et ne peuvent plus mener aucune autre activité rémunératrice. Enfin, ils souhaitent bénéficier d'une bonne formation et un recyclage sur les différentes maladies, leurs causes et les soins appropriés. 


\section{RESULTATS CLES AU NIVEAU DES PRESTATAIRES}

- L'ampleur des infections génito-pelviennes et des IST au sein de la population selon les prestataires. Aussi bien les prestataires professionnels que communautaires reconnaissent que les infections pelviennes et surtout les IST sont fréquentes dans leur zone ; celles-ci frappent les femmes et les hommes de 15 à 49 ans.

\section{Les conséquences des IST se résument selon les prestataires en catégories :}

- les conséquences médicales (rongement des parties génitales, la stérilité, les dispareunies)

- les conséquences sociales de par les disputes que provoquent les IST en cas d'infection d'un des partenaires ou en cas de stérilité ; cela peut avoir comme conséquence la répudiation de la femme.

- les conséquences économiques liées aux dépenses occasionnées par les soins.

- Tandis que les prestataires cliniques perçoivent leur rôle dans un cadre global de traitement curatif et préventif, les prestataires communautaires perçoivent leur rôle dans la prévention conscientisation de leur communauté sur l'existence et les conséquences des IST.

- Les mesures préventives préconisées par les prestataires (cliniques et communautaires) s'articulent autour des changements de comportements sexuels par l'abstinence, la fidélité et l'utilisation des préservatifs.

\section{Pour le cas particulier de la prise en charge du SIDA deux tendances se dégagent :}

- la première tendance est une disposition particulièrement positive et compatissante vis à vis des malades et qui recommande que l'on s'occupe de ces malades ; cette tendance se partage entre les prestataires cliniques et communautaires.

- la deuxième tendance estime qu'on ne plus rien faire pour le SIDA et par conséquent, il ne faut rien faire.

- Il y a une insuffisance dans la formation en matière de prise en charge des pathologies sexuellement transmissibles et le SIDA tant au niveau des prestataires cliniques que communautaires.

- Pour une meilleure prise en charge des IST, les prestataires proposent qu'on leur dispense des formations et des équipements adéquats (laboratoire, matériel IEC)

- La communication sur le thème des infections génito-pelviennes et surtout des IST est peu ouverte au sein de la communauté car considérées comme des maladies honteuses et des vagabondages sexuels ; Les prestataires sont disposés à parler à leurs clients sur le thème des IGP/IST mais se trouvent confrontés au problème de vocabulaire approprié à utiliser. 


\section{CONCLUSION ET RECOMMANDATIONS}

\section{Conclusion $\mathbf{n}^{\circ} \mathbf{1}$}

La communauté a une connaissance de l'existence des maladies pelviennes ; cependant, les causes sont souvent attribuées à des facteurs socioculturels divers. Les jeunes de moins de 25 ans, surtout les filles, ont une ignorance des IST et de leurs conséquences sur leur vie future. Pourtant c'est la tranche qui est la plus exposée aux IST et qui est la plus apte à changer de comportement.

\section{Recommandation $n^{\circ} 1$}

- La mise en place d'un programme de services de santé de la reproduction destinés aux jeunes du milieu rural.

- La décentralisation des activités du Service Jeune de la DRS de Ouagadougou et de la DSF (chargée de l'orientation de ce type d'activité) vers le milieu rural doit être effective dans le cadre du LSC.

- La création d'un cadre de concertation entre jeunes du milieu urbain et rural. Il aboutira à l'élaboration de messages spécifiques en direction des groupes cibles. Ils traiteront notamment des causes exactes des IGP/IST, de leur mode de transmission, des conséquences et des mesures thérapeutiques et préventives adéquates. L'organisation et l'animation de telles rencontres seront assurées par les professionnels de la DSF, la DRSO et plus spécifiquement des districts sanitaires de Kombissiri et de Saponé.

- L'élaboration et la diffusion des messages au sein de la communauté ciblant les jeunes par la formation et l'utilisation de pairs éducateurs.

La recommandation 1 pourrait prendre la forme d'une recherche opérationnelle, passant par la collecte de données de base suivi de l'intervention constituées par les pairs éducateurs.

\section{Conclusion $\mathbf{n}^{\circ} 2$}

La prise en charge des IST devraient se faire à deux niveaux : au niveau communautaire par la sensibilisation par des agents de services à base communautaire et au niveau des formations sanitaires par la prise en charge intégrée (traitement médical et le counseling); de plus la prise en charge devrait prendre la dimension participation de l'homme en traitant le couple.

La communication autour des infections pelviennes et particulièrement des infections sexuellement transmissible pose un problème à plus d'un titre :

- ces pathologies apparaissent comme des maladies honteuses ou le résultats d'un vagabondage sexuel; de ce fait la communauté en discute rarement entre elle.

- du fait que ce sont des maladies dites «honteuses », la consultation ne fait souvent qu'au stade avancé de la maladie

- au sein du couple, lorsque la femme souffre d'une IST, elle a des difficultés à amener son partenaire à se faire traiter pour les raisons précédemment citées et au risque d'être répudiée.

\section{Recommandation $n^{\circ} 2$}

- Au niveau communautaire : il s'agira de former et de recycler les agents de services à base communautaire pour continuer à servir de personnes relais pour offrir des services d'information, d'éducation et de communication à l'intention de leur communauté respective. Ces services se feront à travers des visites à domiciles et des causeries de groupe et des références au sein des formations des personnes souffrant ou présentant des symptômes d'infection génito-pelviennes. Les messages utiliseront au maximum les vocabulaires usité pour designer les pathologies génitopelviennes.

- Au niveau des formations sanitaires : le personnel de santé devrait multiplier les causeries et le counseling sur les IST/SIDA a l'intention des clients fréquentant les FS. Egalement ici, le personnel de santé devraient utilise au maximum les termes connus au sein de la communauté pour designer ces pathologies. 


\section{Conclusion $\mathbf{n}^{\circ} \mathbf{3}$}

L'itinéraire thérapeutique est polymorphe. En effet, il implique le traitement moderne, le traitement traditionnel et religieux par les prières. Cependant force est de reconnaître que les malades commencent par un traitement traditionnel et c'est souvent au prix d'un échec que ceux-ci se présentent dans une formation sanitaire avec des lésions importantes. L'automédication est également de fréquent recours par utilisation de médicaments vendus sur la place du marché.

Le personnel de santé et les prestataires communautaires sont disposés à mener des activités en matière de prévention et de traitement des IGP/IST. Cependant, tous déplorent le manque de matériel approprié pour ces activités mais aussi et surtout la carence en matière de formation dans le domaine. De plus, ceux-ci ne semblent pas à l'aise pour discuter avec les clients sur les infections sexuellement transmissibles, surtout quand il s'agit d'une suspicion de cas de SIDA.

\section{Recommandation $n^{\circ} 3$}

- Former/recycler les professionnels de la santé sur les IST/SIDA et IGP. L'organisation de ces sessions de formation sera assurée par les ECD respectives avec le soutien de la DSF et Population Council. Le contenu de cette activité sera élaboré en tenant compte des résultats de la présente étude.

- Intégrer la prise en charge effective des IST/SIDA dans les formations sanitaires de la zone du LSC.

- Doter les trois centres médicaux de la zone du LSC (Saponé, Kombissiri et Tanghin-Dassouri) de matériels et équipements nécessaires pour le diagnostic et le traitement des cas de IST/SIDA.

Cette dernière recommandation pourrait également revêtir la forme d'une recherche opérationnelle avec un volet intervention par la formation, l'équipement et l'intégration des services IST/VIH/SIDA.

En vue de la mise en œuvre de l'ensemble de ces recommandations et d'un plan d'utilisation effective des résultats de cette étude, il est impératif qu'une table restreinte et une dissémination communautaire soient organisées. 


\section{ANNEXES}




\section{Annexe A : Vocabulaire utilisé par la communauté pour désigner les morbidités de la reproduction}

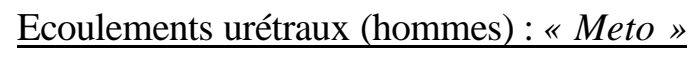

La plupart des enquêtés pensent que ces écoulements ne constituent pas une maladie. Certaines femmes de 25 à 40 ans pensent que les écoulements constituent une maladie pouvant être due au fait qu'un homme ait eu des rapports sexuels avec une femme sur laquelle le mari légitime a mis un piège " bindogo ». Pour certains hommes de 25-40 ans l'écoulement urétral n'est pas une maladie fréquente et elle n'est pas contagieuse.

$\underline{\text { Règles douloureuses : «Silgre » ou «Pekre sin wat wuusguin zabdin » }}$

Selon les hommes de 25-40 ans cette maladie touche les femmes de 25 à 40 ans parce qu'elles ont des activités sexuelles intenses. Les femmes de 25-40 ans disent aussi que la maladie était autrefois liée à l'accouchement ; mais aujourd'hui elle prend de l'ampleur parce que même les jeunes filles qui n'ont pas encore accouché la connaissent, de même que les femmes âgées qui n'en ont pas souffert à leur jeune âge .

$\underline{\text { Pertes blanches : «Kopelga } »}$

C'est un écoulement du sexe de la femme d'un liquide blanc, qui devient rouge au moment des règles ; ce qui signifie pour ces femmes de 25-40 ans que c'est un phénomène normal du cycle de la femme. Cette affection n'est pas connue des filles de moins de 25 ans et des hommes.

Herpès : "Yu sen yit raolem bi poglem » ou «Warpoussi »

Il a été noté que les enquêtés dans l'ensemble ne connaissaient pas l'herpès. Certains hommes et femmes qui semblaient le connaître, l'assimile à un symptôme des prurits.

$\underline{\text { Gonococcie : «Sompissi » ou «Villigaga» }}$

Deux types de «sompissi » ont été décrits par les hommes de 25-40 ans ; selon eux il existe le "sompissi » blanc et le «sompissi » rouge. La maladie peut attraper aussi bien l'homme que la femme. Les hommes sont les plus touchés, particulièrement ceux de 20 à 30 ans qui ont vécu ou qui vivent dans les grandes villes. Les femmes de plus de 40 ans disent c'est une maladie étrangère fréquemment rencontrée en Côte d'Ivoire. Certains estiment que la gonococcie est une maladie qui n'existe plus alors que d'autres soutiennent que la maladie est d'apparition récente. Par contre les femmes de 25-40 ans estiment que la gonococcie est plus fréquente chez les hommes mais ce sont les femmes qui en portent le germe. C'est un mal dont l'ampleur a fortement baissé.

VIH/SIDA : "Ban winga», "Ye wangui», "San koudgo", "Sang baanga», "Weog baanga », «Lag mintaare baanga », «Nonglom baanga », ou « Kan koenga »

Tous les enquêtés disent que le SIDA est une maladie qui attaque aussi bien l'homme que la femme et n'a pas de remède. Les femmes de plus de 40 ans estiment que le SIDA est une maladie des villes comme Ouagadougou ; elles ajoutent que "les jeunes sont les plus concernés parce qu'ils vivent comme des chiens » estimant que les rapports sexuels se font au hasard. Les filles de moins de 25 ans disent que la maladie touche surtout les jeunes et les commerçants ; les jeunes parce qu'ils n'écoutent pas les conseils et les commerçants parce qu'ils tournent beaucoup. Les hommes disent que ce sont les jeunes de 25 à 40 ans et surtout ceux qui reviennent de la Côte d'Ivoire qui sont les plus touchés. Les hommes de 25-40 ans pensent que l'homme est plus vulnérable que la femme ; ils soutiennent que les femmes éliminent les germes du SIDA avec les menstrues. 
Deux formes de prurit ont été décrites :

- Prurits sur tout le corps appelé : «zagongo », «zankada», «koursa-koursa» ou «tiisse»

De façon générale, cette affection touche aussi bien les hommes que les femmes. Les femmes font une différence entre «zagongo » et «zankada»: le «zagongo » ou «koursa-koursa»ou encore "tiisse » désigne le prurit communément connu ; mais le «zankada» est une forme de prurit qui n'existe plus de nos jours. Le «zankada » était une affection qui sévissait en particulier dans les milieux des populations nomades comme les éleveurs Peulhs à cause de leur manque d'hygiène corporelle. Selon les hommes mariés de 25 à 40 ans, le prurit est une maladie mortelle et sexuellement transmissible. Elle peut aussi se transmettre de la mère à l'enfant si la mère en souffre pendant sa grossesse. Les femmes de plus de 40 ans pensent que le «zankada» est une maladie naturelle envoyée par Dieu.

- Prurits se localisant aux organes génitaux externes :

« Goundi », «Kodgo », «Kandga » et « Mende »

Douleurs mictionnelles : «Koom baanga» ou «Koozabdem»

Selon les enquêtés, c'est une affection que contracte aussi bien la femme que l'homme. Mais les hommes de moins de 25 ans distinguent le «koozabdem» qui touche beaucoup plus les vieux et certaines femmes de moins de 25 ans parlent aussi du «koom baanga» qui est fréquent chez les enfants. Il faut souligner que les douleurs mictionnelles sont souvent considérées comme symptômes d'une affection (« sopiisi », «roudsenega », etc.)

$\underline{\text { Règles abondantes : «Koodgo } » \text { ou «Tiiga }}$

Les femmes de 25-40 ans ont dit que les règles abondantes étaient aussi douloureuses. Sur ce point, les hommes de 25-40 ans nous ont aussi parlé des règles fréquentes ("pekre sen wat taw-taw»). Ce sont des affections pas très fréquentes disent-ils (surtout pour les cas du «koodgo» et du «tiiga»).

\section{$\underline{\text { Règles minimes }}$}

Les femmes qui ont été interviewées ne l'ont pas reconnue comme étant une maladie. Pour elles les règles minimes dépendent de l'organisme de chaque femme. Cependant les hommes de moins de 25 ans ont parlé d'une maladie diarrhéique appelée «Coco » (appellation adoptée des Ghanéens) qui peut entraîner les règles minimes (ou abondantes) chez la femme.

\section{Crêtes de coq: «Zig voaka» ou «Norog guenfo »}

Cette maladie n'est pas connue de la plupart des enquêtés. Les hommes de 25-40 ans et les femmes de 25-40 ans pensent la connaitre et disent que c'est une maladie propre aux femmes. Pour certains, il s'agit d' une régénérescence du clitoris après une précédente excision, tandis que les femmes de plus de 40 ans soutiennent le contraire. Néanmoins tous à l'unanimité reconnaissent que c'est une affection rare.

$\underline{\text { Stérilité masculine et féminine : «Yo kiinga » ou «Rabo kieka }}$

La stérilité pour tous les enquêtés est assimilée à un état naturel à l'homme ou à la femme, c'est : l'hermaphrodisme, l'impuissance sexuelle, ou l'absence du trou vaginal chez la femme, d'où les différentes appellations citées. 
Les enquêtés soutiennent que l'adénopathie ne constitue pas une maladie en tant que telle mais qu'elle est l'effet d'une plaie à l'individu. Les hommes de moins de 25 ans disent cependant qu'elle peut être une IST.

Chancre mou : «Tigengenga»ou «Noada»

Ce sont les hommes et les femmes de plus de 40 ans qui ont affirmé connaitre la maladie qui selon eux, concerne aussi bien l'homme que la femme. Certains hommes de 25-40 ans disent que l'affection est propre aux femmes.

Syphilis : «Touma» ou «Pou peelem baanga»

Tous les enquêtés soutiennent le fait que cette maladie n'existe plus de nos jours. Elle a fait des ravages comme le fait le Sida aujourd'hui, disent-ils. La syphilis était prise pour une maladie congénitale (héréditaire) et attaquait beaucoup plus les femmes, nous confient les femmes de plus de 40 ans. Cependant certains hommes de 25-40 ans soutiennent que la maladie existe de nos jours et est une IST.

Morpion : «Karense», «Pen karense » ou «Kin karense»

Selon les enquêtés c'est une affection qui touche aussi bien la femme que l'homme. Les hommes de 25-40 ans l'ont aussi appelée «zankada» (comme pour les prurits) et ceux de moins de 25 ans l'ont assimilée au « touma » (syphilis).

Bilarziose : «Roudsenega» ou «Roudguirse »

C'est une maladie qui a été citée comme IPG par la plupart des enquêtés, qui disent qu'elle touche l'homme et la femme mais est plus fréquente chez les jeunes et les enfants. Les enquêtés affirment aussi qu'elle n'est plus très fréquente de nos jours.

Hydrocèle : "Pouanga », "Poug pouanga »ou «Poonsé »

La plupart des enquêtés (sauf les filles qui disent ne pas disposer d'informations ) disent que tout être humain est prédisposé à avoir la maladie, car tous en portent le germe. Les femmes de 25-40 ans ajoutent que toute femme capable de procréer est exposée au «poug pouanga » ou au «poonsé » spécifiques aux femmes.

\section{$\underline{\text { Hernie : «Kouiga » }}$}

Hernie ou «Kouiga»: Présence d'un renflement au niveau de la région pelvienne, douloureuse à certains moments, fréquente chez les hommes de plus de 40 ans. Ce n'est pas une maladie contagieuse pour la plupart des groupes interviewés. Pour les femmes de plus de 40 ans, c'est « une maladie d'hommes ». Les causes sont essentiellement : la consommation excessive de piment ou d'eau sale, ou encore le travail intensif. Les conséquences couramment évoquées sont la mort et la stérilité. Les soins modernes peuvent soulager le malade en attendant une ablation. 


\section{« Free listing » ou listage libre}

Le but du listage libre est d'obtenir une compréhension du vocabulaire utilisé par la communauté pour décrire les morbidités de la reproduction. L'exercice de listage libre a regroupé 15 femmes issues de la communauté et d'une variété de milieux socioculturels. Il a été demandé à ces femmes sélectionnées de faire la liste de tous les termes connus pour décrire les morbidités de la reproduction et les symptômes associés à ces morbidités connues dans leurs communautés. Les résultats de cet exercice ont été utilisés pour l'élaboration des lignes directrices pour les groupes de discussions dirigées et les entretiens individuels approfondis. Afin de permettre aux agents de collecte de s'imprégner des définitions des morbidités de la reproduction en langue Mooré, il a été fait appel aux services d'un traducteur (Français-Mooré) de l'Institut National de l'Alphabétisation (INA).

\section{Groupes de discussions dirigées (GDD)}

Au total 10 groupes de discussions dirigées ont été réalisés. Le but est d'obtenir une image large de la compréhension des membres de la communauté sur les morbidités de la reproduction. Ils ont été organisés par groupe d'âge et par sexe pour obtenir une homogénéité à l'intérieur de chaque groupe et encourager une discussion ouverte. Les GDD ont porté sur les questions décrites dans les objectifs de l'étude décrits plus haut. Un guide d'entretien a permis de bien focaliser les discussions sur lesdits objectifs. Les groupes avec lesquels les GDD ont eu lieu avaient entre huit et douze participants. L'équipe de collecte avait été organisée de telle sorte que ce soit un homme (animateur) qui anime les discussions avec les hommes et que ce soit une femme (animatrice) qui anime celles avec les femmes. Les données ont été enregistrées sur cassettes audio et des rapports par groupes ont été élaborés.

\section{Entretiens individuels approfondis}

Des entretiens ont été réalisés avec des représentants de différents groupes au sein de la communauté afin d'avoir une bonne connaissance des différentes expériences et perceptions dans la zone. Hommes, femmes, personnel des formations sanitaires et agents des services communautaires ont été interviewés. Les lignes directrices des entretiens se sont basées sur les expériences acquises lors du listage libre et des GDD. Tous les entretiens ont aussi été enregistrés sur cassettes audio ; des rapports sont également disponibles.

\section{Tri par piles}

La méthode du «tri par piles» a été utilisée pour clarifier les croyances sur la relative gravité et les types de comportements de recherche de la santé associés aux différentes morbidités de la reproduction. Une quinzaine des 20 morbidités et symptômes les plus généralement cités ont été sélectionnés sur la base des résultats de la collecte de données et inscrits sur des cartes. Ensuite, il a été demandé à des groupes de 20 hommes et 20 femmes pris au hasard, de regrouper et classer ces cartes en piles selon des critères tels que : les plus fréquents dans la communauté, les sexes concernés, la gravité, l'itinéraire thérapeutique.

\section{Problèmes rencontrés dans la collecte des données}

On observera d'abord le fait que les filles de moins de 25 ans ont été peu loquaces malgré la dextérité des animatrices des GDD. Peu d'informations ont été tirées de ce groupe d'âge féminin. Une grande gêne persistait à l'évocation des IGP. La réponse qui revenait le plus souvent est : "Je ne sais pas ». La situation matrimoniale (célibataire) a constitué une entrave parce qu'on a craint de se faire taxer par certains membres de la communauté de vagabond sexuel ou simplement "d'expérimenté en matière de sexualité ». L'échantillon des filles rencontrées avait pour la plupart entre 14 et 17 ans. Il a été difficile de trouver des filles célibataires. En effet il est rare de rencontrer en milieu rural des filles de 18 ans non encore mariées. Par conséquent le groupe de filles d'âge compris entre 18 et 25 ans n'est pas représenté. L'arrivée de la saison des pluies a failli compromettre les sorties de terrain. Il a fallu le tact et l'efficacité de certains prestataires pour nous permettre d'arriver à bout de la mission avec seulement un jour de retard sur le programme initial. 


\section{Annexe C : Détail descriptif des IGP/IST par les prestataires}

\section{Prestataires professionnels}

1. Les leucorrhées sont liées aux MST (comme la gonococcie). Elles se manifestent par : des pertes de couleur trouble, verdâtres ou jaunâtres (anormales) ou blanches (normales); les pertes sentent mauvais, une sécrétion laiteuse épaisse.

2. Les prurits vulvaires sont liés aux IST et dûs à certains microbes appelés candida. Ils peuvent être également être dûs au manque d'hygiène et sont contagieux. Ils se manifestent par des boutons et des plaies qui apparaissent sur les petites lèvres, sur la vulve et parfois à l'intérieur du vagin.

3. Blennorragie ou gonococcie ou « soumpiissi ». C'est une IST due à un germe appelé gonocoque. Elle peut être aussi causée par : L'utilisation des objets souillés par un malade, quand on urine au même endroit qu'un malade. Pus au début et à la fin des mictions. Maux de bas ventre. Douleurs mictionnelles. Ecoulement urétral (pus blanc). Tuméfaction des organes génitaux chez l'homme. Chez la femme les signes sont sournois et passent inaperçus. Pertes blanches avec une odeur piquante, des douleurs au moment des rapports sexuels et des démangeaisons au niveau de l'appareil génital.

4. La syphilis est une IST due aux germes appelés Treponem. Elle se manifeste de la manière suivante : des maux de ventre, des plaies indolores et propres bien circonscrites (première phase). Disparition des plaies à la deuxième phase, trouble au niveau des organes internes (polakiurie, envie fréquente d'uriner). La troisième se manifeste par des troubles dans l'organisme, troubles cutanées, troubles psychiques et folie.

5. Les candidoses sont dues à un germe appelé candida albicans. Elles se manifestent par des démangeaisons au niveau de la vulve accompagnées de pertes chez la femme. Quant à l'homme, elles se traduisent par des picotements au niveau de l'urètre.

6. Les dysménorrhées sont liées aux règles douloureuses et se manifestent par des douleurs au niveau du bas ventre, débutent chez certaines femmes dès les premières règles.

7. Les douleurs pelviennes sont liées à la grossesse chez la femme et sont de causes inconnues chez l'homme. Elles se manifestent chez la femme par des douleurs au bas ventre et chez l'homme par des douleurs mictionnelles et écoulements urétraux.

8. Les chéloïdes, la stérilité, les pertes blanches, les annexcites, les infections urinaires et les démangeaisons seraient des IPG. Cependant, aucune autre information n'a été donnée sur ces infections.

9. Le chancre mou est une IST. Elle commence par un chancre (dermatose bizarre) au niveau du sexe puis devient une plaie sale.

10. La crête de coq pousse comme un champignon sur le sexe. Sur les causes de ce mal, nous n'avons pas eu d'informations.

11. La bilharziose est due à un microbe appelé bilhazi qui se trouve dans l'eau sale. La baignade dans cette eau sale ou sa consommation donne la maladie. Elle se manifeste par une hématurie terminale. C'est-à-dire qu'au moment de l'émission des urines, il n'y a rien au début, mais à la fin, il y a du sang qui accompagne les urines. Elle donne aussi des douleurs mictionnelles.

12. Le SIDA est une IST. Il se manifeste par une diarrhée de plus d'un mois accompagné de démangeaisons et d'amaigrissement, d'une chute de cheveux. Si pour la plupart des prestataires 
rencontrés, le SIDA n'a pas de remède et est une maladie mortelle, Il a été entendu une fois que le remède existerait chez le «blanc » mais n'est pas encore arrivé ici en Afrique.

\section{Prestataires non professionnels}

1. Le SIDA se manifeste par une diarrhée, des boutons sur le corps, une déshydratation du corps suivi d'un amaigrissement. C'est une maladie due aux rapports sexuels pour les uns et une maladie causée par la prostitution pour les autres. Aucun remède n'existe pour le moment.

2. Prurit adulte («Zaguengo ») se manifeste par un grattage au niveau du sexe de l'homme et de la femme.

3. Le « soumpiissi » ou chaude pisse ou encore gonococcie est une IST. Elle peut s'attraper : quand on urine au même endroit qu'un malade, quand on enjambe un endroit infecté par les urines d'un malade, quand on marche dans certains endroits fréquentés par des animaux tels que les moutons, les chiens (parce qu'à l'origine le « soumpiissi » se rencontrait chez ces animaux seulement). Le vagabondage sexuel est aussi une cause. Les urines sont blanchâtres et ressemblent à du pus. Le malade a des douleurs mictionnelles et un écoulement de liquide blanc gluant suivi de l'émission d'un ver blanchâtre qu'on enlève en l'enroulant autour d'un brin d'herbe. Grattage du sexe provoquant l'éruption de boutons ou saignement.

4. Dysménorrhées sont des douleurs au niveau du bas ventre.

5. Douleurs pelviennes ou «pen zabré » se manifestent chez par des douleurs au bas ventre.

6. Douleurs mictionnelles : dues à la consommation d'eau d'origines diverses (puits, forage, marigot).

7. Démangeaisons au niveau du pelvis sont une IST. Sensation de démangeaison au niveau du pubis.

8. Bilharziose ou « roudsenega » est due à la consommation et baignade d'eau sale. C'est aussi une IST. Le malade a des douleurs mictionnelles et un écoulement de sang à la fin de la miction.

9. Enflure du pelvis : les causes sont ignorées. La principale manifestation est l'enflure du pelvis qui se remplie de pus, ce qui nécessite une incision de la zone.

10. Plaies sur le sexe est une IST. Une plaie apparaît sur le sexe et peut s'agrandir et ronger tout le sexe s'il n'y a pas de traitement.

11. «Kouiga» : Nous n'avons pas eu d'information sur sa cause. Il a été dit qu'il se manifeste par de vives douleurs au niveau du bas ventre, impossibilité d'uriner. Le malade se débat et crie de douleurs.

12. Crête de coq: "C'est une maladie de femme ».

13. «Touma » ou syphilis : Eruption de boutons au niveau du sexe tant chez l'homme que chez la femme. Maladie rare ou inexistante de nos jours. N'existe plus depuis 1946.

14. «Zim lobdb banga» ou maladie des pêcheurs: Infections uro-génitales s'apparentant au roudsenega. Frappe surtout les pêcheurs. L'eau insalubre est la source de contamination. 


\section{Annexe D : Quelques propos retenus}

«Si on insiste sur les conseils, on arrive toujours par les accepter. »

«Quand on parle de SIDA la population s'intéresse plus à l'aspect clinique qu'à la prévention et quand tu n'es pas prudent en la matière, tu vas parler et ils vont prendre les symptômes afficher à un individu et ça crée des problèmes. Il faut donc être souple et dire seulement que le mal existe et qu'il est mortel et dire comment se prémunir».

«J'ai entendu parler du SIDA mais je n'en ai jamais vu. Cependant, si je dis que je n'ai jamais vu un cas de SIDA dans la zone ici, je mens. Le SIDA existe à Toudou ; seulement, la population ne veut pas le reconnaître. Le SIDA ici est assimilé à un sort jeté sur quelqu'un ».

«Ceux qui ont connu des MST sont plus exposés au SIDA. Par exemple, pour le cas de la syphilis, il y a des plaies qui peuvent être des voies par lesquelles le virus du SIDA passe. »

« Je ne peux pas savoir comme ça que quelqu'un souffre de SIDA. Selon ce qu'on nous a enseigné, je ne le sais que lorsque la personne est dans une phase avancée. Malheureusement en ce moment là, je ne peux plus faire quoi que ce soit. » 
Annexe E : Résultats du tri par piles

\begin{tabular}{|c|c|c|c|c|c|}
\hline$?$ & Morbidités & $\begin{array}{c}\operatorname{Sexe}(\mathbf{s}) \\
\text { concerné(s) }\end{array}$ & Gravité & Fréquence & $\begin{array}{c}\text { Type / } \\
\text { comportement de } \\
\text { recherche de santé }\end{array}$ \\
\hline 1 & Roudsinnega & $\begin{array}{l}\text { Hommes \& } \\
\text { femmes }\end{array}$ & $\begin{array}{l}\text { - Très douloureux } \\
\text { au moment des } \\
\text { urines mais } \\
\text { n'empêche pas de } \\
\text { travailler, de } \\
\text { marcher. }\end{array}$ & $\begin{array}{l}\text { - Fréquent au } \\
\text { moment de la } \\
\text { saison des pluies. } \\
\text { - Plus fréquent } \\
\text { chez les hommes. }\end{array}$ & $\begin{array}{l}\text { - Tremper de } \\
\text { l'arachide dans l'eau } \\
\text { pendant longtemps et } \\
\text { boire l'eau. } \\
\text { - Branche \& racine à } \\
\text { faire bouillir et boire } \\
\text { la décoction. }\end{array}$ \\
\hline 2 & Touma & $\begin{array}{l}\text { Hommes \& } \\
\text { femmes }\end{array}$ & $\begin{array}{l}\text { - Douloureux, très } \\
\text { grave parce qu'il y } \\
\text { a des plaies et tue. }\end{array}$ & N'existe plus. & $\begin{array}{l}\text { - Utiliser un épi de } \\
\text { maïs pour gratter et } \\
\text { appliquer un produit } \\
\text { (qu'ils ne connaissent } \\
\text { pas ) }\end{array}$ \\
\hline 3 & Soumpissi & $\begin{array}{l}\text { Hommes \& } \\
\text { femmes }\end{array}$ & $\begin{array}{l}\text { - Très douloureux } \\
\text { et tue. }\end{array}$ & $\begin{array}{l}\text { - Pas fréquent. } \\
\text { - Il existe quelques } \\
\text { cas rares ( } 2 \text { à } 3 \\
\text { personnes) }\end{array}$ & $\begin{array}{l}\text { - Couper du citron en } \\
\text { tranches, faire bouillir } \\
\text { et boire. } \\
\text { - Faire bouillir des } \\
\text { feuilles de gouiga et } \\
\text { boire. }\end{array}$ \\
\hline 4 & Zagongo & $\begin{array}{l}\text { Hommes \& } \\
\text { femmes }\end{array}$ & $\begin{array}{l}\text { - Pas très grave, il } \\
\text { ne tue pas, ça } \\
\text { ronge les ongles à } \\
\text { cause du grattage. }\end{array}$ & $\begin{array}{l}\text { - Fréquent chez } \\
\text { les femmes, rare } \\
\text { chez les hommes. }\end{array}$ & $\begin{array}{l}\text { - Produit liquide qu'on } \\
\text { verse sur le corps. } \\
\text { - Poudre qu'on met } \\
\text { dans les yeux et avec } \\
\text { un système de } \\
\text { balayage on fait } \\
\text { tomber les germes. }\end{array}$ \\
\hline 5 & Couina & $\begin{array}{l}\text { Hommes \& } \\
\text { femmes }\end{array}$ & $\begin{array}{l}\text { - Le kouigraka et } \\
\text { le kouigrouko sont } \\
\text { graves. }\end{array}$ & $\begin{array}{l}\text { - Chacun naît avec } \\
\text { son kouiga. }\end{array}$ & $\begin{array}{l}\text { - Aller au dispensaire } \\
\text { pour une intervention } \\
\text { chirurgicale. }\end{array}$ \\
\hline 6 & Zankada & $\begin{array}{l}\text { Hommes \& } \\
\text { femmes }\end{array}$ & $\begin{array}{l}\text {-Avant, c'était une } \\
\text { maladie très grave } \\
\text { mais aujourd'hui } \\
\text { elle n'existe plus. }\end{array}$ & $\begin{array}{l}\text { - N'existe plus } \\
\text { aujourd'hui. } \\
\text { - Avant c'était } \\
\text { fréquent. }\end{array}$ & $\begin{array}{l}\text { - Savon traditionnel } \\
\text { pour se laver. } \\
\text { - Utiliser la potasse } \\
\text { pour se frotter. }\end{array}$ \\
\hline 7 & Kinn karansé & $\begin{array}{l}\text { Hommes \& } \\
\text { femmes }\end{array}$ & $\begin{array}{l}\text { - Très grave parce } \\
\text { que c'est honteux. }\end{array}$ & $\begin{array}{l}\text { - N'est plus } \\
\text { fréquent } \\
\text { aujourd'hui à } \\
\text { cause des } \\
\text { conditions } \\
\text { d'hygiène qui sont } \\
\text { meilleures. } \\
\end{array}$ & $\begin{array}{l}\text { - Utiliser du pétrole. } \\
\text { - Piler une plante et } \\
\text { frotter sur le sexe. }\end{array}$ \\
\hline 8 & Poug pouanga & $\begin{array}{l}\text { Hommes \& } \\
\text { femmes }\end{array}$ & $\begin{array}{l}\text { - Grave parce que } \\
\text { c'est difficile de } \\
\text { faire les selles. } \\
\text { - Déchire l'anus. }\end{array}$ & $\begin{array}{l}\text { - Pas très } \\
\text { fréquent, quelques } \\
\text { cas. }\end{array}$ & $\begin{array}{l}\text {-Faire bouillir des } \\
\text { écorces de Gouïga et } \\
\text { se purger avec la } \\
\text { décoction. }\end{array}$ \\
\hline
\end{tabular}




\begin{tabular}{|c|c|c|c|c|c|}
\hline$?$ & Morbidités & $\begin{array}{c}\operatorname{Sexe}(\mathbf{s}) \\
\text { concerné(s) }\end{array}$ & Gravité & Fréquence & $\begin{array}{c}\text { Type / } \\
\text { comportement de } \\
\text { recherche de santé }\end{array}$ \\
\hline 9 & Koodgo & Femmes & $\begin{array}{l}\text { - C'est douloureux } \\
\text { mais pas très } \\
\text { grave. } \\
\text { - Si c'est un sort } \\
\text { jeté, c'est grave } \\
\text { parce que le sang } \\
\text { coule sans cesse. }\end{array}$ & $\begin{array}{l}\text { - Pour certains } \\
\text { c'est fréquent } \\
\text { cycle } \\
\text { menstruel. } \\
\text { - Pour d'autres ce } \\
\text { n'est pas fréquent. }\end{array}$ & $\begin{array}{l}\text { - Remède pas } \\
\text { vulgaire.Il faut avoir } \\
\text { la maladie pour en } \\
\text { demander le remède. }\end{array}$ \\
\hline 10 & Sida & $\begin{array}{l}\text { Hommes \& } \\
\text { femmes }\end{array}$ & $\begin{array}{l}\text { - Très grave parce } \\
\text { que ça détruit les } \\
\text { intestins, il tue } \\
\text { aussi et il n'y a pas } \\
\text { de remède. }\end{array}$ & Fréquent. & $\begin{array}{l}\text { - Pas de remède. } \\
\text { - Se maintenir. } \\
\text { - Utiliser la capote. }\end{array}$ \\
\hline 11 & Pouanga & $\begin{array}{l}\text { - Pour certains ce } \\
\text { sont uniquement les } \\
\text { hommes. } \\
\text { - Pour d'autres les } \\
\text { femmes aussi en } \\
\text { souffrent. }\end{array}$ & $\begin{array}{l}\text {-Pas grave } \\
\text {,douloureux quand } \\
\text { ça commence à } \\
\text { pousser. } \\
\text { - Non douloureux } \\
\text { quand ça devient } \\
\text { visible. }\end{array}$ & $\begin{array}{l}\text { - Fréquent chez } \\
\text { les hommes. } \\
\text { Rare chez les } \\
\text { femmes. }\end{array}$ & $\begin{array}{l}\text { - Faire bouillir des } \\
\text { racines et boire pour } \\
\text { que le pus coule. } \\
\text { - C'est ce que le } \\
\text { blanc appelle } \\
\text { «opération ». }\end{array}$ \\
\hline 12 & Kandga & $\begin{array}{l}\text { Hommes \& } \\
\text { femmes }\end{array}$ & $\begin{array}{l}\text { - Très grave parce } \\
\text { que douloureux et } \\
\text { peut détruire le } \\
\text { sexe et les reins. }\end{array}$ & - Pas fréquent. & $\begin{array}{l}\text { - Brûler des racines } \\
\text { et frotter. } \\
\text { - Frotter du beurre de } \\
\text { karité pour calmer la } \\
\text { douleur. }\end{array}$ \\
\hline 13 & Kossenkogo. & $\begin{array}{l}\text { Hommes \& } \\
\text { femmes }\end{array}$ & $\begin{array}{l}\text { - Grave chez la } \\
\text { femme parce qu'il } \\
\text { y'a risque } \\
\text { d'avortement ou } \\
\text { de naissance } \\
\text { prématurée. }\end{array}$ & - Pas fréquent. & $\begin{array}{l}\text { - Voir chez les vieilles } \\
\text { femmes qui disposent } \\
\text { du produit. }\end{array}$ \\
\hline 14 & Koom. & NSP & NSP & NSP & NSP \\
\hline 15 & Pougou & $\begin{array}{l}\text { Hommes \& } \\
\text { femmes }\end{array}$ & $\begin{array}{l}\text { - Douloureux. } \\
\text { - Chez certains il } \\
\text { y'a une infection } \\
\text { et il faut inciser. }\end{array}$ & $\begin{array}{l}\text { - Pas fréquent } \\
\text { - Survient lorsqu'il } \\
\text { y'a une plaie. }\end{array}$ & $\begin{array}{l}\text { - Frotter la potasse. } \\
\text { - Brûler des racines } \\
\text { et frotter. } \\
\text { - Piler du tinkouitikoui } \\
\text { et frotter. } \\
\text { - Beurre de karité. }\end{array}$ \\
\hline 16 & Kiissé & Femmes & $\begin{array}{l}\text { - C'est grave } \\
\text { parce que, peut } \\
\text { provoquer } \\
\text { des avortements. } \\
\text { - Stérilité. } \\
\end{array}$ & $\begin{array}{l}\text { - Fréquent chez } \\
\text { les femmes. }\end{array}$ & $\begin{array}{l}\text { - Aller chez les } \\
\text { vieilles femmes pour } \\
\text { l'enlever. }\end{array}$ \\
\hline 17 & $\begin{array}{l}\text { Règles } \\
\text { douloureuses }\end{array}$ & Femmes & $\begin{array}{l}\text { - C'est fréquent } \\
\text { mais les femmes } \\
\text { n'en parlent pas. }\end{array}$ & $\begin{array}{l}\text {-Les gens n'en } \\
\text { parlent pas donc } \\
\text { ils disent ne pas } \\
\text { savoir si c'est } \\
\text { fréquent ou pas. }\end{array}$ & $\begin{array}{l}\text { - Le remède existe } \\
\text { - Traditionnel mais, } \\
\text { comme on ne parle } \\
\text { pas de la maladie de } \\
\text { façon vulgaire, ils } \\
\text { disent ne pas savoir } \\
\text { comment il se fait / } \\
\text { constitution. }\end{array}$ \\
\hline
\end{tabular}




\begin{tabular}{|c|l|l|l|l|l|}
\hline$?$ & Morbidités & \multicolumn{1}{c|}{$\begin{array}{c}\text { Sexe(s) } \\
\text { concerné(s) }\end{array}$} & Gravité & \multicolumn{1}{|c|}{ Fréquence } & $\begin{array}{c}\text { Type / } \\
\text { comportement de } \\
\text { recherche de santé }\end{array}$ \\
\hline 18 & $\begin{array}{l}\text { Règles minimes } \\
\text { (kuivigo) }\end{array}$ & Femmes & $\begin{array}{l}\text {-Pas grave parce } \\
\text { qu'il n'empêche } \\
\text { pas de dormir. }\end{array}$ & $\begin{array}{l}\text { - Aujourd'hui c'est } \\
\text { fréquent mais les } \\
\text { femmes n'en } \\
\text { parlent pas. }\end{array}$ & $\begin{array}{l}\text { - Produit liquide à } \\
\text { boire. }\end{array}$ \\
\hline
\end{tabular}

Zagongo: Ils distinguent trois types de Zagongo.

\begin{tabular}{|l|l|}
\hline Morbidité & Symptômes \\
\hline Zagongo simple: & Démangeaisons simples. \\
\hline Soulga (araignée ) & Due à la piqûre d'une araignée, démange tout le corps. \\
\hline Goundi & $\begin{array}{l}\text { Type de démangeaison qui infecte une partie spécifique du corps: soit le } \\
\text { sexe - la jambe - les mains. }\end{array}$ \\
\hline
\end{tabular}

Kouiga: Le groupe distingue 5 types de Kouiga.

\begin{tabular}{|l|l|}
\hline Morbidité & Symptômes \\
\hline Kouigmouka & Se traduit par un manque de virilité. \\
\hline Kouigraga & $\begin{array}{l}\text { Se traduit par un enflement du bas ventre. On peut ressentir des douleurs } \\
\text { au niveau du genou, du bras aussi. }\end{array}$ \\
\hline Kouiyanga & Pas douloureux. Pas de manifestations particulières. \\
\hline Kouigrouko & $\begin{array}{l}\text { Se présente comme une marmite sur un foyer. Il faut une intervention } \\
\text { pour l'enlever. }\end{array}$ \\
\hline Kougpouga & $\begin{array}{l}\text { Se traduit par un enflement du ventre si la personne consomme des } \\
\text { aliments qui ne lui conviennent pas, comme haricot, spaghetti, tô. }\end{array}$ \\
\hline
\end{tabular}

